Collection SFN 11 (2010) 43-69

(C) Owned by the authors, published by EDP Sciences, 2010

DOI: $10.1051 / \mathrm{sfn} / 201011004$

\title{
Bonnes pratiques de la diffusion de neutrons aux petits angles
}

\author{
D. Lairez
}

\author{
Laboratoire Léon Brillouin, CEA-CNRS UMR 12, CEA Saclay, 91191 Gif-sur-Yvette Cedex, \\ France \\ e-mail : lairez@cea.fr
}

Résumé. Conceptuellement, une expérience de diffusion de rayonnement donne accès à la densité spectrale de puissance de la densité de longueur de diffusion d'un échantillon (ou section efficace différentielle de diffusion). Cette grandeur s'exprime en $\mathrm{cm}^{2}$.

Ce cours se veut un guide pratique et explicatif de la réalisation d'une expérience sur un spectromètre de diffusion de neutrons aux petits angles. Dans un premier temps le vocabulaire est précisé. Les notions de cohérence du faisceau en relation avec la résolution instrumentale sont abordées. Puis sont exposées les idées générales devant guider l'expérimentateur dans le choix des réglages du spectromètre. Sont exposées ensuite les mesures, qui outre celle de la diffusion de l'échantillon, doivent être réalisées pour extraire l'information souhaitée des données collectées: mesure de la transmission; mesure permettant la normalisation des spectres par l'efficacité du détecteur; mesure des différentes contributions qui s'ajoutent au spectre inhérent à l'échantillon; étalonnage du spectromètre permettant d'exprimer la mesure en unité absolue; etc. Très souvent, l'expérimentateur ne s'intéresse qu'à la contribution due aux corrélations des fluctuations du contraste local dans l'échantillon. Aux petits angles, les fluctuations de densité ou celles dites « incohérentes » s'apparentent alors à un bruit de fond inhérent à l'échantillon. Nous discuterons de la façon d'évaluer et de soustraire ces contributions.

\section{Table des matières}

\section{Introduction}

1 Ce qui est mesuré idéalement $\quad \mathbf{4 5}$

1.1 Neutron et onde associée . . . . . . . . . . . . . . . . . . . . . . . . . . 45

1.2 Diffusion par un atome $\ldots \ldots \ldots \ldots \ldots \ldots$

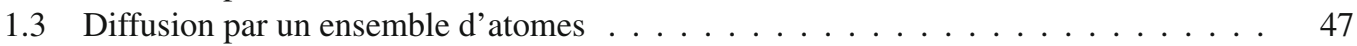

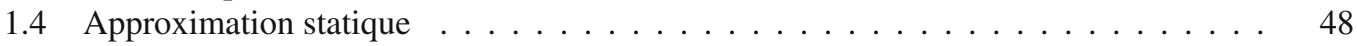

1.5 Remarques sur la fonction de corrélation et sa transformée de Fourier . . . . . . . . 49

1.5.1 Un milieu homogène ne diffuse pas . . . . . . . . . . . . . . . . . . . . . . 49

1.5.2 Longueur de corrélation très courte . . . . . . . . . . . . . . . . 49

2 Spectromètre $\quad \mathbf{5 0}$

2.1 Schéma d'un spectromètre . . . . . . . . . . . . . . . . . . 50

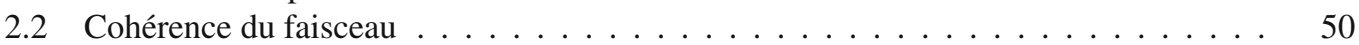

2.3 Volume de cohérence et volume diffusant . . . . . . . . . . . . . . . 51

2.4 Choix de la collimation ......................... 52

This is an Open Access article distributed under the terms of the Creative Commons Attribution-Noncommercial License 3.0, which permits unrestricted use, distribution, and reproduction in any noncommercial medium, provided the original work is properly cited. 
2.5 Choix de la configuration $\ldots \ldots \ldots \ldots \ldots \ldots \ldots$

3 Soustraction des différentes contributions à la mesure $\quad \mathbf{5 5}$

3.1 Approximation $«$ petits angles $» \ldots \ldots \ldots \ldots \ldots$

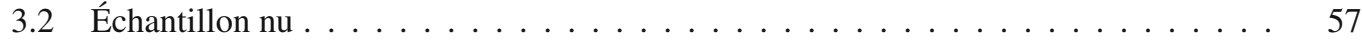

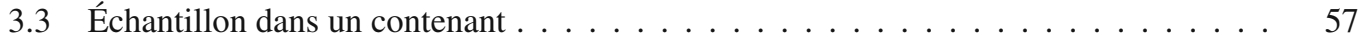

3.4 Correction $u$ grands angles $» \ldots \ldots \ldots \ldots \ldots \ldots \ldots$

4 Normalisation des spectres $\quad 59$

4.1 Diffuseur de référence . . . . . . . . . . . . . . . . . . . . . 59

4.2 Gagner du temps et de la précision . . . . . . . . . . . . . . . . . . 60

4.3 Importance des corrections $\ll$ grands angles $» \ldots \ldots \ldots \ldots$. . . . . . . . . 61

5 Étalonnage du spectromètre et mesures absolues $\quad 61$

5.1 Quel est le problème $\quad \ldots \ldots \ldots \ldots \ldots$. . . . . . . . . . . . . . 61

5.2 Comment faire des mesures en unité absolue $\quad \ldots \ldots \ldots \ldots$. . . . . . . . . . 62

5.3 À quoi sert l'étalonnage $\quad \ldots \ldots \ldots \ldots \ldots \ldots$

6 Aspects relatifs aux échantillons $\quad 62$

6.1 Diffusion incohérente d'un neutron par les atomes . . . . . . . . . . . . . 62

6.2 Diffusion incohérente par un mélange de composés chimiques élémentaires . . . . . . 64

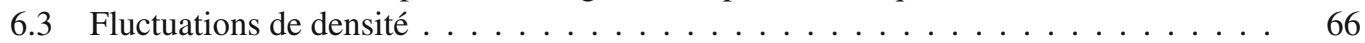

6.4 «Incohérent» de mélange . . . . . . . . . . . . . . . 66

6.5 Comment évaluer le bruit de fond inhérent à l'échantillon ? . . . . . . . . . . . . 67

6.6 Quelle épaisseur pour l'échantillon ? . . . . . . . . . . . . . . . . 68

$\begin{array}{lc}\text { Conclusion } & 68\end{array}$

\section{Introduction}

Les expériences de diffusion de neutrons aux petits angles, comme toutes celles menées auprès de grands instruments, laissent finalement peu de temps à la formation « sur le tas » et comparées aux expériences plus légères demandent une préparation accrue. Même si elles sont encadrées par un spécialiste de l'instrument utilisé, le maximum d'efficacité sera obtenu par cette préparation en amont. Elle permettra en particulier une bonne compréhension des différentes phases de l'expérience et son véritable « pilotage » par l'expérimentateur lui-même qui est le seul à en connaître tous les enjeux. Le but premier de ce cours est de faciliter cette préparation.

La diffusion de neutrons est assez peu enseignée dans les cursus classiques. Aussi le premier obstacle rencontré par un expérimentateur qui aborde ces techniques est celui du langage. La première partie de ce cours est donc consacrée à clarifier le vocabulaire et préciser ce dont il s'agira par la suite. Pour cela j'ai adopté délibérément le vocabulaire du traitement du signal qui est commun à beaucoup de domaines de la physique et effectué la «traduction » en jargon neutronicien. Les parties suivantes traitent des problèmes pratiques dans l'ordre où ils se présentent lors de la conduite d'une expérience. Ainsi la partie 2 est consacrée aux réglages du spectromètre et notamment aux critères qui doivent guider les choix qui s'offrent à l'expérimentateur. Les parties 3 et 4 abordent ensuite le traitement des données afin de préciser a priori toutes les mesures à réaliser lors de l'expérience. La partie 5 concerne les mesures complémentaires permettant l'étalonnage du spectromètre. Enfin, la dernière partie est consacrée à certains aspects relatifs aux échantillons, principalement à son bruit de fond inhérent et à la façon de le mesurer. 
Tableau 1. Table des symboles classés (approximativement) par ordre d'apparition.

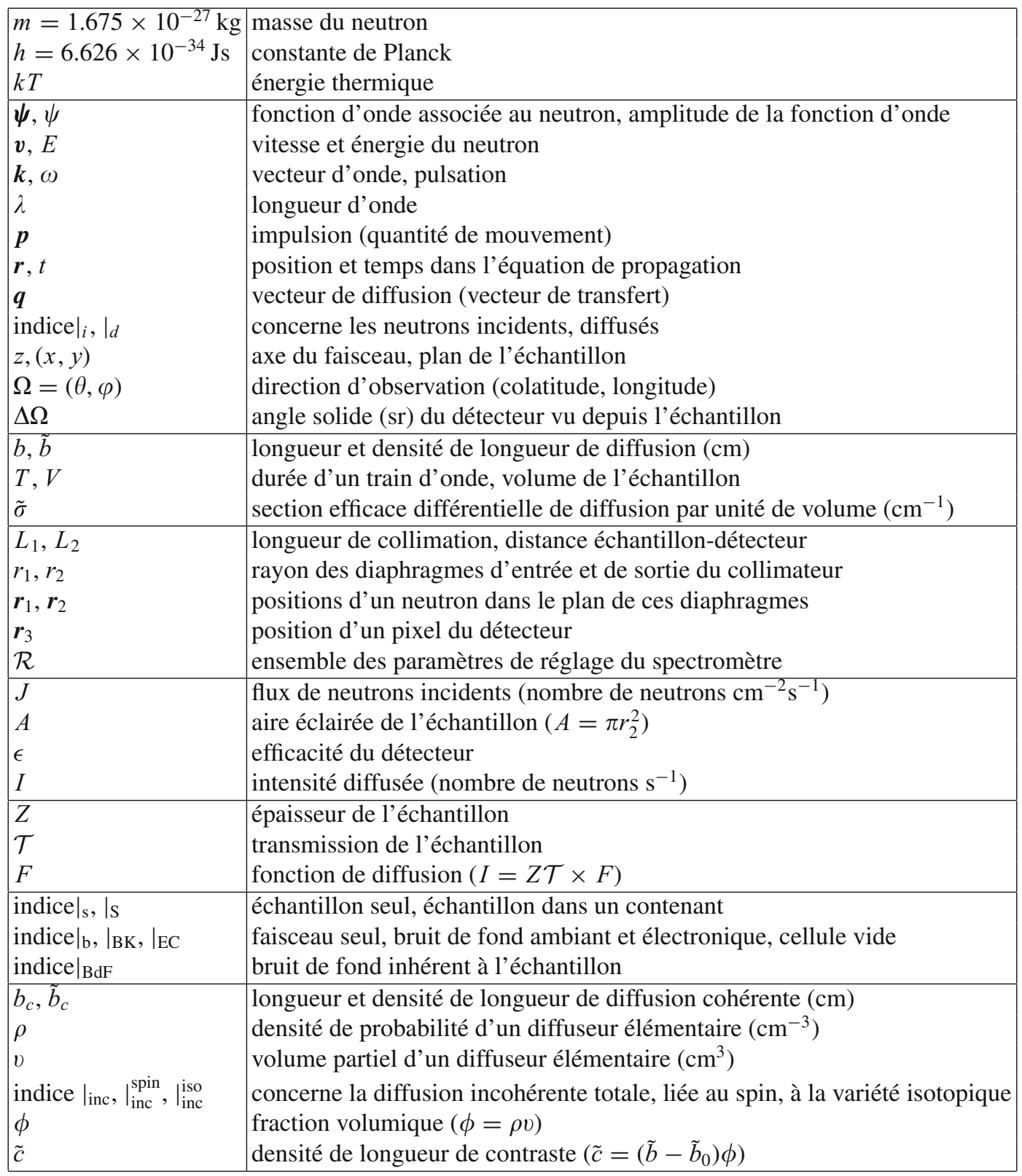

\section{CE QUI EST MESURÉ IDÉALEMENT}

\subsection{Neutron et onde associée}

Les mesures de diffusion de neutrons présentent toutes les caractéristiques observées habituellement sur les figures d'interférences des ondes. Pour les expliquer, la mécanique quantique associe à un neutron de vitesse $\boldsymbol{v}$ et d'énergie $E=m v^{2} / 2$ une fonction d'onde $\psi$ (ou amplitude de probabilité) dont l'équation 
de propagation est :

$$
\psi(\boldsymbol{r}, t)=\psi e^{i(\omega t-k \boldsymbol{r})}
$$

et dont la pulsation $\omega$ et le vecteur d'onde $\boldsymbol{k}$ sont

$$
\omega=E / \hbar ; \quad \boldsymbol{k}=m \boldsymbol{v} / \hbar
$$

avec $m$ la masse du neutron et $\hbar=h / 2 \pi$. Pour une vitesse de l'ordre de $400 \mathrm{~m} / \mathrm{s}$, soit à peine supérieure à celle du son dans l'air, la longueur d'onde $\lambda=h / m v$ est typiquement de l'ordre de $10 \AA$.

Le terme « amplitude de probabilité » pour $\psi$ signifie que le module carré $|\psi(\boldsymbol{r}, t)|^{2}=\psi(\boldsymbol{r}, t) \boldsymbol{\psi}^{*}(\boldsymbol{r}, t)$ est la densité de probabilité de détecter la particule en $\boldsymbol{r}$ à l'instant $t$ (grandeur homogène à un nombre de neutrons par unité de volume). Des neutrons se déplaçant à la même vitesse ont la même fonction d'onde et en particulier la même phase. Ce faisceau monocinétique est représenté par une somme de fonctions d'onde monochromatiques dont l'équation de propagation est la même que l'Eq. 1.1 et d'amplitude $\psi$ telle que :

$$
J=v \times|\psi|^{2}=\frac{\hbar k}{m} \times|\psi|^{2}
$$

où $J$ est le flux de neutrons (nombre de neutrons par unité d'aire et de temps). Les détecteurs comptent des neutrons et mesurent donc ce flux plutôt que l'amplitude de l'onde elle-même.

\subsection{Diffusion par un atome}

Soit une onde plane incidente $\boldsymbol{\psi}_{i}$ de vecteur $\boldsymbol{k}_{i}$ et de pulsation $\omega_{i}$. En rencontrant un atome, cette onde est en partie réfléchie de façon diffuse également dans toutes les directions. L'onde diffusée $\psi_{d}$ est sphérique et son amplitude décroît comme l'inverse de la distance d'observation $r_{\mathrm{obs}}$ (la densité de probabilité $\left|\psi_{d}\right|^{2}$ décroît comme $1 / r_{\text {obs }}^{2}$ ). Très loin de l'atome diffuseur, $\psi_{d}$ pourra être considérée localement comme une onde plane de vecteur $\boldsymbol{k}_{d}$ et de pulsation $\omega_{d}$. Si l'interaction entre l'onde et l'atome se produit à l'instant $t$ en un lieu $\boldsymbol{r}$ de l'échantillon, la phase de l'onde incidente est $\omega_{i} t-\boldsymbol{k}_{i} \boldsymbol{r}$. A celle-ci s'ajoute le déphasage dû au parcours de l'onde diffusée entre le lieu d'interaction et celui d'observation: $\omega_{d}\left(t_{\mathrm{obs}}-t\right)-\boldsymbol{k}_{d}\left(\boldsymbol{r}_{\mathrm{obs}}-\boldsymbol{r}\right)$. L'équation de propagation de $\boldsymbol{\psi}_{d}$ s'écrit alors :

$$
\psi_{d}=b \frac{\psi_{i}}{r_{\mathrm{obs}}} e^{i\left(\omega_{d} t_{\mathrm{obs}}-\boldsymbol{k}_{d} \boldsymbol{r}_{\mathrm{obs}}\right)} e^{i\left(\left(\boldsymbol{k}_{d}-\boldsymbol{k}_{i}\right) \boldsymbol{r}-\left(\omega_{d}-\omega_{i}\right) t\right)} .
$$

Le facteur de proportionnalité $b$ est homogène à une longueur et appelé « longueur de diffusion » de l'atome. Cette longueur caractérise la portée des interactions entre le rayonnement et l'atome. En ne retenant dans l'Eq. 1.4 que les variables $\boldsymbol{r}$ et $t$ relatives aux coordonnées de l'interaction du rayonnement avec l'atome, on peut écrire :

$$
\psi_{d}(\boldsymbol{r}, t)=\psi_{d}(0,0) e^{i(\boldsymbol{q} \boldsymbol{r}-\omega t)} \quad \text { avec } \quad\left\{\begin{array}{l}
\boldsymbol{q}=\boldsymbol{k}_{d}-\boldsymbol{k}_{i} \\
\omega=\omega_{d}-\omega_{i}
\end{array} .\right.
$$

Le vecteur $\boldsymbol{q}$ est appelé « vecteur de diffusion » ou « transfert de quantité de mouvement » car $\hbar \boldsymbol{q}$ représente le transfert d'impulsion (ou de quantité de mouvement) de l'atome à la particule associée à l'onde diffusée, tandis que $\hbar \omega$ est l'énergie de ce transfert.

Un détecteur d'aire $s$ enregistrera une intensité diffusée $I$ (exprimée en nombre de neutrons par unité de temps) égale à :

$$
I=v_{d}\left|\psi_{d}\right|^{2} s=J \frac{v_{d}}{v_{i}} b^{2} \Delta \Omega
$$

où $\Delta \Omega=s / r_{\mathrm{obs}}^{2}$ est l'angle solide que forme le détecteur vu depuis l'atome et $v_{d}$ la vitesse des neutrons diffusés. 
Lorsque plusieurs atomes diffusent, l'intensité n'est pas toujours uniforme sur la sphère entourant l'échantillon et peut dépendre de l'orientation $\Omega$ de l'angle solide d'observation. Il est nécessaire alors de considérer l'intensité $d I$ diffusée dans la direction $\Omega$ par unité d'angle solide $d \Omega$. Cette quantité est en général rapportée au flux de neutrons incidents, soit :

$$
\frac{d I / d \Omega}{J}=\frac{v_{d}\left|\psi_{d}\right|^{2}}{v_{i}\left|\psi_{i}\right|^{2}} r_{\mathrm{obs}}^{2}=\frac{k_{d}}{k_{i}} b^{2}
$$

$(d I / d \Omega) / J$ est homogène à une aire et appelée « section efficace différentielle » de diffusion.

Implicitement nous avons considéré une onde diffusée ayant une pulsation bien définie, ce qui n'est jamais le cas. Un transfert d'énergie entre l'onde et l'atome diffuseur entraîne une distribution de la pulsation. Cette distribution est responsable d'un battement de l'onde résultante qui est l'analogue temporel des interférences. L'onde interceptée par un détecteur loin de l'échantillon n'est plus une onde plane idéale mais un train d'onde dont la durée $T$ est d'autant plus réduite que la distribution est large. Chaque composante de Fourier $\psi_{d}(\omega)$ de l'onde diffusée s'écrit :

$$
\psi_{d}(\omega)=\int_{T} \psi_{d}(t) e^{-i \omega t} d t
$$

Le nombre total de neutrons de ce train d'onde $\int\left|\psi_{d}(t)\right|^{2} d t$ s'exprime également dans le domaine fréquentiel comme $\frac{1}{2 \pi} \int\left|\psi_{d}(\omega)\right|^{2} d \omega$ (théorème de Parseval). La grandeur $\left|\psi_{d}(\omega)\right|^{2} / 2 \pi$ est la densité spectrale d'énergie de l'onde diffusée. On utilise plutôt $\left|\psi_{d}(\omega)\right|^{2} / T$, soit la densité spectrale de puissance de l'onde diffusée qui représente le nombre de neutrons par unité de volume dont l'énergie est comprise entre $\hbar(\omega-d \omega)$ et $\hbar(\omega+d \omega)$, c'est à dire un nombre de neutrons par unité de volume et de fréquence. L'équation 1.7 devient :

$$
\frac{d I(\omega) / d \Omega}{J}=\frac{v_{d}\left|\psi_{d}(\omega)\right|^{2} / T}{v_{i}\left|\psi_{i}\right|^{2}} r_{\mathrm{obs}}^{2} .
$$

La grandeur $(d I(\omega) / d \Omega) / J$ est homogène à une aire par unité de fréquence et est appelée « section efficace différentielle partielle de diffusion ».

\subsection{Diffusion par un ensemble d'atomes}

Pour un échantillon constitué de $N$ atomes, si les ondes diffusées par chacun d'eux ne le sont qu'une seule fois, l'onde diffusée totale à un instant $t$ donné résulte de leur superposition :

$$
\psi_{d}=\frac{\psi_{i}}{r_{\mathrm{obs}}} \sum_{a=1}^{N} b_{a} e^{i\left(\boldsymbol{q} \boldsymbol{r}_{a}-\omega t\right)} .
$$

Plutôt que de considérer les atomes de l'échantillon, il est plus commode d'envisager ses éléments de volume. La somme discrète est alors remplacée par l'intégrale :

$$
\psi_{d}(\boldsymbol{q}, t)=\frac{\psi_{i}}{r_{\mathrm{obs}}} e^{-i \omega t} \int_{V} \tilde{b}(\boldsymbol{r}, t) e^{i(\boldsymbol{q} \boldsymbol{r})} d^{3} \boldsymbol{r}
$$

où $\tilde{b}(\boldsymbol{r}, t)$ est appelée « densité de longueur de diffusion » (autrement dit $\tilde{b}(\boldsymbol{r}, t) d^{3} r$ est la longueur de diffusion de l'élément de volume $d^{3} r$ au point $r$ à l'instant $t$ ). L'intégrale dans l'Eq. (1.11) décrit les interférences entre les ondes diffusées par les différents éléments de volume de l'échantillon. En fonction du vecteur de diffusion $\boldsymbol{q}$, l'amplitude de $\psi_{d}$ varie selon que ces ondes élémentaires sont en phase ou non. L'équation (1.11) exprime que l'onde diffusée est la transformée de Fourier spatiale de la densité de longueur de diffusion, le vecteur $\boldsymbol{q}$ étant la variable conjuguée de $\boldsymbol{r}$. 
Dans le domaine temporel, de façon similaire à l'Eq. (1.8), chaque composante de Fourier $\boldsymbol{\psi}_{d}(\boldsymbol{q}, \omega)$ de l'onde diffusée s'écrit :

$$
\boldsymbol{\psi}_{d}(\boldsymbol{q}, \omega)=\frac{\psi_{i}}{r_{\mathrm{obs}}} \int_{T} \int_{V} \tilde{b}(\boldsymbol{r}, t) e^{i(\boldsymbol{q} \boldsymbol{r}-\omega t)} d^{3} \boldsymbol{r} d t=\frac{\psi_{i}}{r_{\mathrm{obs}}} \mathrm{TF}(\tilde{b}(\boldsymbol{r}, t))
$$

où on note $\operatorname{TF}(\tilde{b}(\boldsymbol{r}, t))$ la transformée de Fourier spatiale et temporelle de la densité de longueur de diffusion. Finalement la section efficace différentielle partielle de diffusion (Eq. 1.9) s'écrit :

$$
\frac{d I(\boldsymbol{q}, \omega) / d \Omega}{J}=\frac{1}{2 \pi} \frac{k_{d}}{k_{i}} \frac{|\operatorname{TF}(\tilde{b}(\boldsymbol{r}, t))|^{2}}{T} .
$$

On rapporte en général cette grandeur au volume $V$ de l'échantillon et on note :

$$
\tilde{\sigma}(\boldsymbol{q}, \omega)=\frac{d I(\boldsymbol{q}, \omega) / d \Omega}{J V}=\frac{1}{2 \pi} \frac{k_{d}}{k_{i}} \frac{|\mathrm{TF}(\tilde{b}(\boldsymbol{r}, t))|^{2}}{V T} .
$$

La grandeur $\tilde{\sigma}(\boldsymbol{q}, \omega)$ est appelée « section efficace différentielle partielle de diffusion par unité de volume ». Elle est homogène à l'inverse d'une longueur par unité de fréquence et est proportionelle à la densité spectrale de puissance de $\tilde{b}(\boldsymbol{r}, t)$ que le théorème de Wiener-Khintchine relie à la fonction d'autocorrélation $\langle\tilde{b}(0,0) \tilde{b}(\boldsymbol{r}, t)\rangle$ :

$$
\frac{|\operatorname{TF}(\tilde{b}(\boldsymbol{r}, t))|^{2}}{V T}=\operatorname{TF}\langle\tilde{b}(0,0) \tilde{b}(\boldsymbol{r}, t)\rangle .
$$

Les crochets \langle\rangle signifient une moyenne sur toutes les origines possibles dans l'espace et le temps du produit $\tilde{b}(0,0) \tilde{b}(\boldsymbol{r}, t)$. Finalement :

$$
\tilde{\sigma}(\boldsymbol{q}, \omega)=\frac{1}{2 \pi} \frac{k_{d}}{k_{i}} \operatorname{TF}\langle\tilde{b}(0,0) \tilde{b}(\boldsymbol{r}, t)\rangle .
$$

Cette fonction d'autocorrélation, ou ses diverses transformées de Fourier, est la grandeur essentielle à laquelle idéalement une expérience de diffusion de rayonnement donne accès. Elle représente la probabilité qu'ayant une certaine valeur en un point de l'échantillon et à l'instant initial, la densité (de longueur de diffusion) soit identique à une distance $r$ au bout d'un temps $t$. Les variables expérimentales $\boldsymbol{q}$ et $\omega$ sont les variables conjuguées de $\boldsymbol{r}$ et $t$ qui permettent de décrire l'échantillon dans l'espace direct. Pour une interprétation rapide et intuitive des expériences, $q^{-1}$ et $\omega^{-1}$ peuvent être vus comme les échelles dans l'espace et le temps à laquelle l'échantillon est observé.

\subsection{Approximation statique}

Une expérience de diffusion de neutrons aux petits angles consiste à utiliser un faisceau incident monochromatique et à enregistrer le nombre de neutrons diffusés selon une direction $\Omega=(\theta, \varphi)$ donnée, où $\theta$ et $\varphi$ sont les coordonnées sphériques usuelles. Soit $(d I(\Omega) / d \Omega) / J V$ l'intensité mesurée rapportée au flux de neutrons incidents, à l'unité d'angle solide et au volume de l'échantillon. Cette grandeur intègre toute la distribution en énergie des neutrons diffusés :

$$
\frac{d I(\Omega) / d \Omega}{J V}=\int \tilde{\sigma}(\Omega, \omega) \epsilon\left(\omega_{i}+\omega\right) d \omega
$$

où $\epsilon\left(\omega_{i}+\omega\right)$ est l'efficacité du détecteur qui dépend de l'énergie des neutrons. Si $\tau_{R}$ est le temps de relaxation moyen d'une fluctuation de densité de longueur de diffusion, le transfert d'énergie moyen entre le neutron et l'échantillon est de l'ordre de $\Delta E \approx h / \tau_{R}$. L'effet de la distribution en énergie des neutrons pourra être négligé si $\Delta E$ est faible comparée à l'énergie moyenne $E_{i}=\frac{1}{2} h v / \lambda$ des neutrons incidents. Ainsi lorsque

$$
\tau_{R} \gg 2 \lambda / v
$$


les diffuseurs élémentaires à l'origine des ondes produisant les interférences pourront être considérés comme immobiles (c'est l'approximation statique) et la diffusion comme élastique (se produisant sans transfert d'énergie). Par exemple, pour $\lambda=10 \AA$, on obtient $2 \lambda / v=5$ ps. La condition 1.18 est donc aisément réalisée lors d'expériences de diffusion aux petits angles qui sont des mesures à basse résolution et pour lesquelles les diffuseurs élémentaires (c'est à dire les détails les plus fins auxquels on accède) sont déjà assez grands. Leur temps caractéristique $\tau_{R}$ augmente beaucoup avec leur taille et devient vite suffisant pour l'approximation statique (par exemple le temps de Zimm d'un segment de chaîne polymère varie comme sa taille au cube). Les pulsations $\omega_{i}$ et $\omega_{d}$ pourront être considérées égales $(\omega=0)$ ainsi que les normes $k_{i}$ et $k_{d}$ des vecteurs d'onde. Dans ce cas, à une direction $\Omega$ correspond un vecteur de diffusion, d'où $I(\Omega) \simeq I(\boldsymbol{q}), \tilde{\sigma}(\Omega, \omega)=\tilde{\sigma}(\boldsymbol{q}, \omega)$ et $\epsilon\left(\omega_{i}\right)=$ Cst. L'équation 1.17 donne :

$$
\frac{d I(\Omega) / d \Omega}{J V} \simeq \frac{d I(\boldsymbol{q}) / d \Omega}{J V} \simeq \epsilon\left(\omega_{i}\right) \tilde{\sigma}(q) \quad \text { avec } \quad \tilde{\sigma}(q)=\operatorname{TF}\langle\tilde{b}(0) \cdot \tilde{b}(\boldsymbol{r})\rangle
$$

$\tilde{\sigma}(q)$ est la « section efficace différentielle de diffusion par unité de volume » et est homogène à l'inverse d'une longueur. Dans cette approximation, la norme du vecteur de diffusion est :

$$
q=\frac{4 \pi}{\lambda} \sin \left(\frac{\theta}{2}\right)
$$

où $\theta$ est l'angle que forme les vecteurs d'onde incidente et diffusée.

\subsection{Remarques sur la fonction de corrélation et sa transformée de Fourier}

\subsubsection{Un milieu homogène ne diffuse pas}

Soit $\tilde{b}$ la densité moyenne de longueur de diffusion du milieu. En un lieu $\boldsymbol{r}$ particulier, la densité de longueur de diffusion peut s'écarter de cette moyenne: $\tilde{b}(\boldsymbol{r})=\tilde{b}+\Delta \tilde{b}$. La fonction de corrélation s'écrit

$$
\langle\tilde{b}(0) \cdot \tilde{b}(\boldsymbol{r})\rangle=\tilde{b}^{2}+\langle\Delta \tilde{b}(0) \cdot \Delta \tilde{b}(\boldsymbol{r})\rangle+\tilde{b}\langle\Delta \tilde{b}(0)+\Delta \tilde{b}(\boldsymbol{r})\rangle .
$$

Par définition, $\Delta \tilde{b}(0)$ et $\Delta \tilde{b}(\boldsymbol{r})$ sont nuls en moyenne, d'où finalement :

$$
\langle\tilde{b}(0) \cdot \tilde{b}(\boldsymbol{r})\rangle=\tilde{b}^{2}+\langle\Delta \tilde{b}(0) \cdot \Delta \tilde{b}(\boldsymbol{r})\rangle .
$$

Le premier terme de cette expression est une constante. Sa transformée de Fourier ne donne qu'une contribution à $q=0$ qui n'est pas mesurable car confondue avec le faisceau transmis à travers l'échantillon. C'est l'équivalent d'une composante continue d'un signal. Finalement l'Eq. 1.19 peut s'écrire :

$$
\tilde{\sigma}(\boldsymbol{q})=\operatorname{TF}\langle\Delta \tilde{b}(0) \cdot \Delta \tilde{b}(\boldsymbol{r})\rangle
$$

Ce qui exprime que seules les fluctuations autour de la valeur moyenne, qui correspondent à une hétérogénéité du milieu, contribuent à sa section efficace.

\subsubsection{Longueur de corrélation très courte}

La technique de diffusion aux petits angles est dédiée à la mesure des fluctuations dont la longueur de corrélation $a$ est très grande comparée aux dimensions atomiques. Toute fluctuation dont la longueur de corrélation serait trop courte pour la résolution de l'appareil verra sa fonction de corrélation perçue comme une distribution $\delta$ de Dirac.

$$
\left\langle\left.\Delta \tilde{b}(0) \cdot \Delta \tilde{b}(\boldsymbol{r})\right|_{r \gg a} \simeq\left\langle\Delta \tilde{b}^{2}\right\rangle \delta(\boldsymbol{r} / a) .\right.
$$




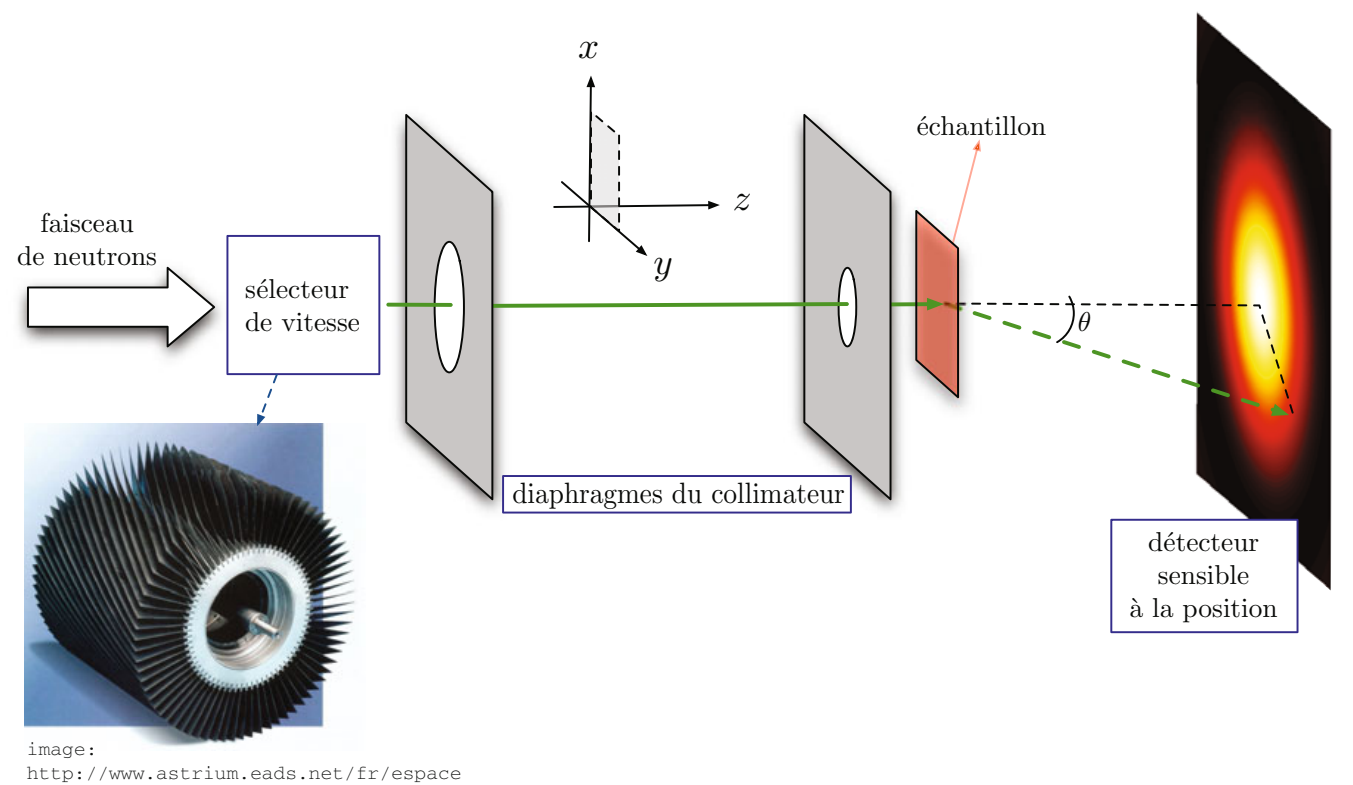

Figure 1. Schéma d'un spectromètre de diffusion de neutrons aux petits angles.

$\left\langle\Delta \tilde{b}^{2}\right\rangle$ est la variance (moyenne des carrés des écarts à la moyenne) de la densité de longueur de diffusion. La transformée de Fourier donne :

$$
\tilde{\sigma}\left(\boldsymbol{q} \ll a^{-1}\right)=\left\langle\Delta \tilde{b}^{2}\right\rangle a^{3} .
$$

Ces fluctuations de longueur de corrélation très courtes se manifestent par un spectre de diffusion indépendant de $q$ qui est l'équivalent du spectre d'un bruit blanc (cf. partie 6).

\section{SPECTROMÈTRE}

\subsection{Schéma d'un spectromètre}

Lors d'une expérience de diffusion de neutrons aux petits angles, un faisceau de neutrons de vitesse et d'incidence données éclaire un échantillon. Les neutrons sont des particules lentes et leur vitesse peut être sélectionnée mécaniquement par un absorbant en rotation dans lequel leur est ménagé un passage hélicoïdal. La divergence du faisceau est contrôlée par les diaphragmes du collimateur. La majeure partie de ces neutrons traverse l'échantillon sans interagir avec les atomes qui le constituent, tandis qu'une fraction des neutrons incidents sont déviés de leur trajectoire. Ces derniers sont responsables d'un halo de diffusion autour du faisceau transmis (cf. Fig. 1). L'expérience consiste à mesurer au moyen d'un détecteur sensible à la position cette diffusion dite «centrale» (ou « aux petits angles ») dont l'analyse fournit des informations sur les propriétés statiques (c'est à dire structurales et thermodynamiques) de l'échantillon.

\subsection{Cohérence du faisceau}

Un aspect fondamental de la dualité onde-particule est donné par la relation d'incertitude entre l'impulsion d'une particule et sa position qui stipule qu'au mieux :

$$
\Delta \boldsymbol{p} \cdot \Delta \boldsymbol{r}=\hbar / 2 .
$$


Ainsi, pour un neutron de vitesse parfaitement connue se déplaçant dans la direction $z$ perpendiculaire au plan $(x y)$ de l'échantillon, les incertitudes sur l'impulsion dans les sens longitudinal et transversal tendent vers 0 . Ce neutron est alors totalement délocalisé $(\Delta x, \Delta y, \Delta z \rightarrow \infty)$ et peut interagir (et donc diffuser) à la fois avec tous les atomes de l'échantillon. L'onde qui lui est associée est une onde plane idéale dont la diffusion produit des interférences. Dans la pratique, il s'agit plutôt d'un paquet d'onde dont les dimensions varient en raison inverse de la largeur chromatique et de la divergence du faisceau et détermine son volume de cohérence.

La sélection mécanique de la vitesse des neutrons incidents entraîne une incertitude relative $\Delta v / v=$ $\Delta \lambda / \lambda$ constante qui ne dépend que de la géométrie du passage hélicoïdal réservé aux neutrons [1]. Pour un neutron donné, cette incertitude détermine celle de son impulsion dans le sens longitudinale de sa propagation: $\left\langle\boldsymbol{p}_{\text {long. }}\right\rangle=\hbar \boldsymbol{k}_{i}, \Delta \boldsymbol{p}_{\text {long. }}=\left\langle\boldsymbol{p}_{\text {long. }}\right\rangle \times \Delta \lambda / \lambda$ et la relation 2.1 donnent :

$$
2 \Delta z=\frac{\lambda}{2 \pi} \times\left(\frac{\Delta \lambda}{\lambda}\right)^{-1} .
$$

L'onde associée à ce neutron est un train d'onde de longueur $2 \Delta z$ typiquement de l'ordre de 1 à $10 \mathrm{~nm}$ selon la résolution $\Delta \lambda / \lambda$. La durée de passage de ce train dans un plan transverse définit un temps de cohérence :

$$
t_{c}=\frac{2 \Delta z}{v}=\omega^{-1} \times\left(\frac{\Delta \lambda}{\lambda}\right)^{-1}
$$

$\omega=2 \pi v / \lambda$ est la pulsation moyenne du train d'onde. Ce temps de cohérence est typiquement de l'ordre de 10 à 100 ps selon la résolution. Il peut avoir une incidence sur les mesures des propriétés dynamiques de l'échantillon. En diffusion de neutrons aux petits angles, les mesures sont réalisées dans le plan $(x y)$ c'est à dire pour des vecteurs de diffusion $\boldsymbol{q} \perp z$. Elles sont donc insensibles aux corrélations de densité de l'échantillon selon la direction $z$ et à la perte de cohérence du faisceau dans cette direction (excepté toutefois l'effet mentionné dans la partie 2.3).

La collimation a pour but de limiter la divergence $\Delta \theta$ du faisceau qui éclaire l'échantillon. Sa valeur non nulle implique une composante transverse de l'impulsion connue à $\Delta \boldsymbol{p}_{\text {trans }}=\Delta \theta \times \hbar \boldsymbol{k}_{i}$ près. La relation 2.1 donne $\Delta x=\Delta y=1 /\left(2 k_{i} \Delta \theta\right)$. Aux petits angles la norme du vecteur de diffusion (Eq. 1.20) peut s'écrire $q \simeq k_{i} \theta$, d'où finalement :

$$
2 \Delta x=2 \Delta y=q^{-1} \times\left(\frac{\Delta \theta}{\theta}\right)^{-1}
$$

$2 \Delta x$ et $2 \Delta y$ sont les dimensions de l'aire de cohérence du faisceau. $\mathrm{Si} q^{-1}$ représente l'échelle de longueur à laquelle les corrélations de l'échantillon sont observées, étant donné que $\Delta \theta / \theta$ est toujours plus petit que 1, l'Eq. 2.4 montre que la cohérence transverse du faisceau est toujours suffisante pour que ce soit effectivement celle de l'échantillon qui soit sondée.

\subsection{Volume de cohérence et volume diffusant}

Le volume $v_{c}=2^{3} \Delta x \Delta y \Delta z$ de cohérence du faisceau est toujours suffisant pour sonder les corrélations de l'échantillon. Toutefois, il reste beaucoup plus petit que le volume diffusant $V$ de l'échantillon. La section efficace mesurée est donc une moyenne des sections efficaces des $V / v_{c}$ volumes de cohérence :

$$
\tilde{\sigma}(\boldsymbol{q})=\frac{1}{V / v_{c}} \sum_{v_{i}=1}^{V / v_{c}} \tilde{\sigma}_{v_{i}}(\boldsymbol{q})=\overline{\operatorname{TF}\langle\tilde{b}(0) \cdot \tilde{b}(\boldsymbol{r})\rangle_{(0, \boldsymbol{r}) \in v_{c}}}
$$

Autrement dit, les $V / v_{c}$ volumes de cohérence sont autant de petits échantillons identiques (sous ensembles de l'échantillon total) dont les spectres de diffusion seraient mesurés indépendamment pour en faire ensuite la moyenne. 


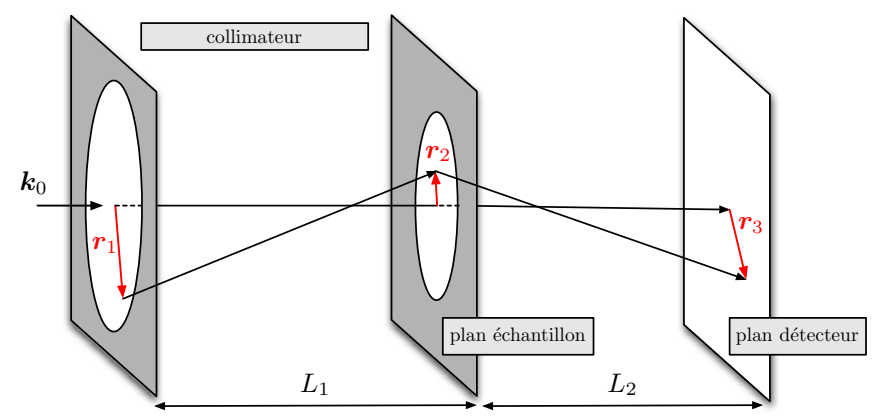

Figure 2. Schéma de principe d'un collimateur.

Par ailleurs, le flux de neutrons éclairant l'échantillon est au mieux de l'ordre de $10^{9} \mathrm{~cm}^{-2} \mathrm{~s}^{-1}$, ce qui signifie un neutron dans l'échantillon toutes les nanosecondes. Ce temps est nettement supérieur au temps de cohérence $t_{c}$ et implique que chaque neutron diffuse « un par un » indépendamment des autres. De façon similaire à l'Eq. (2.5), la section efficace mesurée est donc une moyenne des sections efficaces perçues par chaque neutron.

\subsection{Choix de la collimation}

La collimation a une incidence directe sur la résolution de la mesure [2] et sur le flux de neutrons mesurés. Les neutrons incidents atteignent le plan d'un premier diaphragme du collimateur en un point $\boldsymbol{r}_{1}$ par rapport à son centre (voir Fig. 2). Ils atteignent ensuite un second diaphragme situé au niveau du plan échantillon en un point $\boldsymbol{r}_{2}$. Après diffusion, ils sont détectés en un point $\boldsymbol{r}_{3}$ du détecteur. La distance entre diaphragmes est notée $L_{1}$ et celle entre échantillon et détecteur $L_{2}$. Soit $\boldsymbol{k}_{0}$ le vecteur d'onde dans l'axe du spectromètre, $\boldsymbol{k}_{i}$ le vecteur d'onde réellement incident sur l'échantillon et $\boldsymbol{k}_{d}$ le vecteur d'onde diffusée. On établit les relations suivantes :

$$
\left.\begin{array}{rl}
\boldsymbol{k}_{\mathrm{i}}=\boldsymbol{k}_{0}+\frac{\boldsymbol{r}_{2}-\boldsymbol{r}_{1}}{L_{1}} \\
\boldsymbol{k}_{\mathrm{d}}=\boldsymbol{k}_{0}+\frac{\boldsymbol{r}_{3}-\boldsymbol{r}_{2}}{L_{2}} \\
\boldsymbol{q}=\boldsymbol{k}_{\mathrm{d}}-\boldsymbol{k}_{\mathrm{i}}
\end{array}\right\} \Rightarrow\left\{\begin{aligned}
\frac{\boldsymbol{r}_{3}}{L_{2}}-\boldsymbol{q} & =\frac{\boldsymbol{r}_{2}}{L_{2}}+\frac{\boldsymbol{r}_{2}-\boldsymbol{r}_{1}}{L_{1}} \\
& =\boldsymbol{r}_{2}\left(\frac{1}{L_{1}}+\frac{1}{L_{2}}\right)-\frac{\boldsymbol{r}_{1}}{L_{1}}
\end{aligned}\right.
$$

Les variables aléatoires $\boldsymbol{r}_{1}$ et $\boldsymbol{r}_{2}$ sont indépendantes et leurs variances s'ajoutent pour donner celle de l'angle d'incidence sur le détecteur :

$$
\begin{aligned}
\left\langle\Delta \theta^{2}\right\rangle & =\left\langle\Delta\left(\boldsymbol{r}_{2}\left(\frac{1}{L_{1}}+\frac{1}{L_{2}}\right)\right)^{2}\right\rangle+\left\langle\Delta\left(\frac{\boldsymbol{r}_{1}}{L_{1}}\right)^{2}\right\rangle=\frac{1}{2}\left(r_{2}\left(\frac{1}{L_{1}}+\frac{1}{L_{2}}\right)\right)^{2}+\frac{1}{2}\left(\frac{r_{1}}{L_{1}}\right)^{2} \\
& =\frac{1}{2}\left(\frac{r_{2}}{L_{2}}\right)^{2}\left(\frac{L_{1}+L_{2}}{L_{1}}\right)^{2}+\frac{1}{2}\left(\frac{r_{1}}{L_{1}}\right)^{2}
\end{aligned}
$$

où $r_{1}, r_{2}$ sont les rayons des diaphragmes.

Soit $J_{0}$ le flux du faisceau de neutrons avant collimation et $\theta_{c}$ sa divergence. La résolution angulaire $\left\langle\Delta \theta^{2}\right\rangle^{1 / 2}$ est toujours inférieure à la divergence (sinon la collimation serait inutile). Ce qui implique que parmi toutes les incidences possibles de l'angle solide $\theta_{c}^{2}$ le collimateur prélève celles qui s'incrivent dans l'angle solide $\pi r_{2}^{2} / L_{1}^{2}$. Cela s'applique pour tous les points d'entrée $\boldsymbol{r}_{1}$ des neutrons dans le collimateur, soit une intensité éclairant l'échantillon proportionnelle à $I_{\text {éclairage }} \propto$ $J_{0} \int_{0}^{r_{1}} 2 \pi r\left(\pi r_{2}^{2} / L_{1}^{2}\right) / \theta_{c}^{2} d r \propto J_{0} r_{1}^{2}\left(r_{2} / L_{1}\right)^{2}$. Le flux de neutrons diffusés par unité d'angle solide est quant 


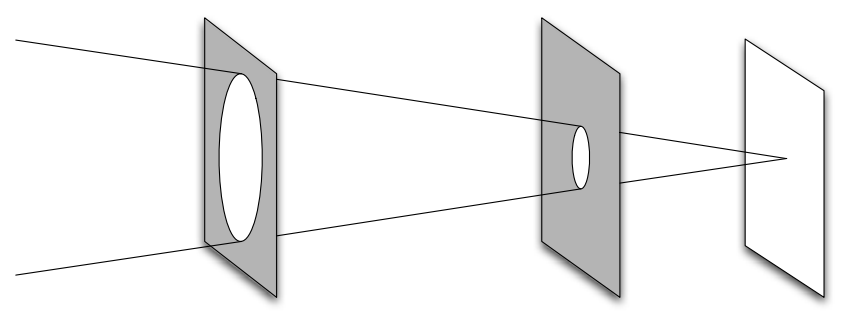

Figure 3. Géométrie optimale du collimateur.

à lui proportionnel à $\mathcal{J}=I_{\text {éclairage }} / L_{2}^{2}$, soit :

$$
\mathcal{J}=J_{0} \times\left(\frac{r_{1}}{L_{1}}\right)^{2} \times\left(\frac{r_{2}}{L_{2}}\right)^{2} .
$$

Pour une résolution donnée, les équations 2.7 et 2.8 donnent les conditions de collimation fournissant le flux maximum. On trouve :

$$
\frac{r_{2}}{L_{2}}=\frac{r_{1}}{L_{1}+L_{2}}
$$

ce qui correspond à la situation où le cône inscrit dans les deux diaphragmes pointe sur le détecteur (Fig. 3). En utilisant les équations 2.8 et 2.9, on montre que dans ces conditions optimales le flux diffusé par unité d'angle solide est $\mathcal{J}_{\text {opt }}=J_{0}\left(r_{2} / L_{2}\right)^{4}\left(1+L_{2} / L_{1}\right)^{2}$, soit :

$$
\mathcal{J}_{\text {opt }}=J_{0} \times\left(\frac{1}{L_{2}}\right)^{4} \times r_{1}^{2} r_{2}^{2} .
$$

Le rayon $r_{2}$ est la taille de la surface éclairée de l'échantillon qui sera limitée par son volume. Le rayon $r_{1}$ sera lui limité par la section du guide de neutrons alimentant le spectromètre. En maximisant dans tous les cas ces deux longueurs, le flux de neutrons est donc proportionnel à $\left(1 / L_{2}\right)^{4}$. Pour une position $\boldsymbol{r}_{3}$ de détection, l'angle de diffusion est $\theta \simeq r_{3} / L_{2}$. Finalement le flux optimum varie comme :

$$
\mathcal{J}_{\text {opt }}(\theta) \propto J_{0} \theta^{4} .
$$

\subsection{Choix de la configuration}

La variable qui rend compte des expériences de diffusion de neutrons aux petits angles est le vecteur de diffusion dont la norme est de l'ordre de $q \simeq 2 \pi \theta / \lambda$. Des valeurs identiques du vecteur de diffusion peuvent être obtenues pour des couples $(\theta, \lambda)$ différents. On entend ici par configuration du spectromètre, les réglages correspondants au choix d'un couple $(\theta, \lambda)$ particulier. Ce choix est guidé par :

1. la variation du flux de neutrons diffusés avec l'angle de diffusion et la collimation, variation donnée par l'Eq. 2.11 en supposant le collimateur dans sa géométrie optimale.

2. la dépendance en longueur d'onde du flux de neutrons incidents $J_{0}(\lambda)$.

3. la dépendance en longueur d'onde de l'efficacité du détecteur $\epsilon(\lambda)$.

Ces contributions étant indépendantes, le flux $\mathcal{J}$ de neutrons mesuré est proportionnel à :

$$
\mathcal{J} \propto \theta^{4} J(\lambda) \epsilon(\lambda) .
$$

Aux petits angles $\theta \simeq \lambda q / 2 \pi$ et le flux varie comme :

$$
\mathcal{J} \propto q^{4} \lambda^{4} J(\lambda) \epsilon(\lambda)
$$

Pour un vecteur de diffusion donné, le flux optimum ne dépend donc que de la longueur d'onde. 
L'efficacité du détecteur varie avec la longueur d'onde comme :

$$
\epsilon(\lambda)=1-e^{-\lambda / \lambda_{c}} .
$$

Par exemple, sur deux des spectromètres de diffusion aux petits angles du LLB (PAXE et PACE), la caractéristique $\lambda_{c}$ est égale à $5 \AA$.

Avant d'être guidés vers un spectromètre de diffusion aux petits angles, les neutrons produits par un réacteur sont ralentis par de multiples chocs inélastiques avec les atomes d'un modérateur à basse température (dit « source froide »). Les neutrons sont ainsi thermalisés et la distribution de leur vitesse obéit à la statistique de Maxwell-Boltzmann d'un gaz parfait à la température $T$ du modérateur: $P(v) \propto v^{2} \exp \left(-m v^{2} / 2 k T\right)$. $P(v)$ est la densité de probabilité qu'un neutron ait la vitesse $v$. Exprimée en fonction de la longueur d'onde $\lambda=h / m v$, on obtient $P(\lambda)=P(v) \times d v / d \lambda \propto$ $\exp \left(-\left(\lambda_{\text {therm }} / \lambda\right)^{2}\right) \times \lambda^{-4}$, où $\lambda_{\text {therm }}=\left(h^{2} / 2 m k T\right)^{1 / 2}$ est la longueur d'onde thermique qui est de l'ordre de $7 \AA$ pour une source froide typique à $20 \mathrm{~K}$. La densité de flux (flux par unité de longueur d'onde) est $d J(\lambda) / d \lambda=v P(v), v$ étant proportionnel à $1 / \lambda$, on obtient :

$$
\frac{d J(\lambda)}{d \lambda} \propto e^{-\left(\lambda_{\text {therm }} / \lambda\right)^{2}} \times \lambda^{-5} .
$$

Si $\Delta \lambda$ est la largeur de l'intervalle des longueurs d'onde sélectionnées par le monochromateur, alors $J(\lambda)=(d J / d \lambda) \Delta \lambda$. Pour un sélecteur mécanique $\Delta \lambda \propto \lambda$, soit $J(\lambda)=(d J / d \lambda) \lambda$. Finalement en fonction de la densité de flux, l'équation 2.13 devient :

$$
\mathcal{J} \propto q^{4} \lambda^{5} \times\left(\frac{d J(\lambda)}{d \lambda}\right) \epsilon(\lambda) .
$$

Le produit $(d J(\lambda) / d \lambda) \epsilon(\lambda)$ est mesurable directement sur le spectromètre par la méthode de temps de vol [1]. Elle consiste à hacher le faisceau incident polychromatique au moyen d'un disque en rotation, absorbant les neutrons et muni d'une fente radiale. Le passage de celle-ci devant le faisceau, déclenche une bouffée et l'enregistrement en fonction du temps (donc de la longueur d'onde) du nombre de neutrons atteignant le détecteur. Le résultat de cette mesure réalisée sur le spectromètre PACE est présenté sur la Fig. 4 qui appelle deux remarques.

1. Pour une source froide dont le modérateur thermaliserait parfaitement les neutrons sans les absorber, la densité de flux est donnée par l'Eq. 2.15. Le flux mesuré pour un vecteur $q$ donné correspondrait alors à la courbe en pointillés de la Fig. 4. L'asymptote de l'Eq. 2.15 est atteinte lentement et en suivant cette courbe dans le domaine usuel des longueurs d'onde de la diffusion aux petits angles, les grandes longueurs d'onde seraient toujours à privilégier dans le choix de la configuration ( $\mathcal{J}$ plus grand).

2. Dans la pratique, la loi de puissance $(d J / d \lambda)_{\lambda \gg \lambda_{\text {term }}} \propto \lambda^{-5}$ de l'équation 2.15 présente une coupure exponentielle. La ligne continue de la Fig. 4 représente le meilleur ajustement du spectre mesuré (points de la Fig. 4) par temps de vol. Il est obtenu en prenant deux températures pour la source froide ( $20 \mathrm{~K}$ et $80 \mathrm{~K}$, pour un meilleur ajustement aux courtes longueurs d'onde et rendre compte d'une thermalisation imparfaite du faisceau) et une fonction de coupure exponentielle de longueur d'onde caractéristique égale à $11 \AA$.

Sur le spectromètre PACE, pour $\lambda \lesssim 10 \AA$ la longueur d'onde la plus grande devra toujours être privilégiée dans le choix d'une configuration et inversement pour les longueurs d'onde supérieures à $10 \AA ̊$. Quel que soit le spectromètre, la variation du flux mesuré à vecteur de diffusion donné et résolution constante sera toujours du même type que celui présenté sur la Fig. 4, même si la valeur optimale de la longueur d'onde peut varier d'un spectromètre à l'autre. 


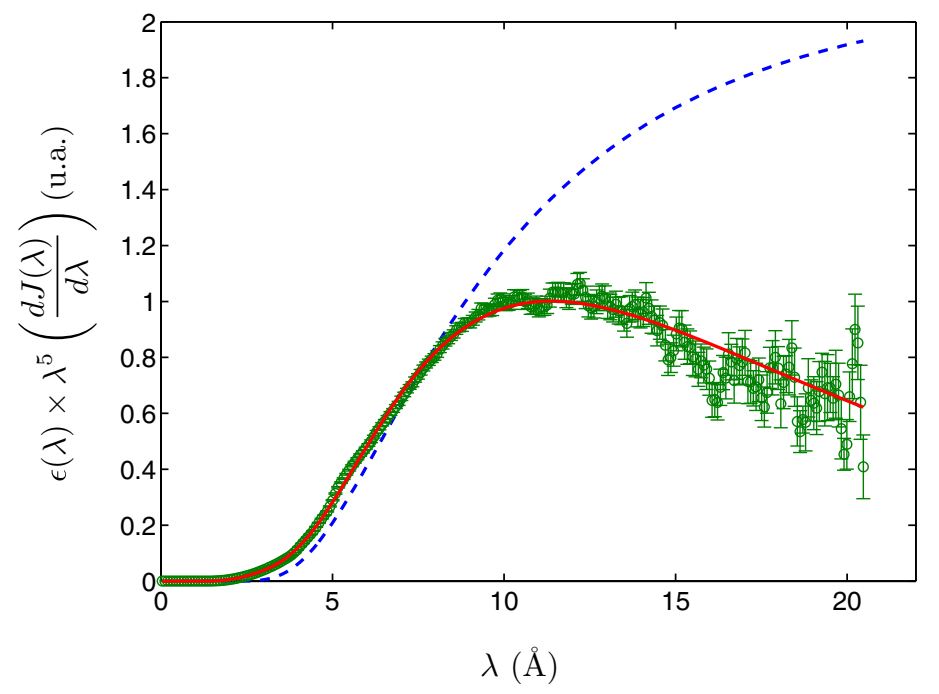

Figure 4. Flux de neutrons diffusés $\mathcal{J} \propto \lambda^{5} \epsilon(\lambda)(d J(\lambda) / d \lambda)$ en fonction de la longueur d'onde. Points: densité de flux mesurée par temps de vol sur le spectromètre PACE (LLB). Ligne pointillée: densité de flux donnée par l'Eq. 2.15 avec une source froide à $20 \mathrm{~K}$. Ligne continue: meilleur ajustement des données obtenu avec deux températures pour la source froide $(20 \mathrm{~K}$ et $80 \mathrm{~K})$ et une fonction de coupure exponentielle de caractéristique égale à $11 \AA ̊$.

\section{SOUSTRACTION DES DIFFÉRENTES CONTRIBUTIONS À LA MESURE}

Le traitement des données de diffusion de neutrons aux petits angles a pour but d'extraire des mesures réalisées pour un échantillon sa section efficace différentielle par unité de volume. Cela demande une attention particulière et des mesures complémentaires à celle de la diffusion de l'échantillon. Pour se convaincre de cet aspect non trivial, il est édifiant de lire les pionniers de cette technique qui démontrent la pertinence de la diffusion centrale qu'ils observent pour un échantillon en la comparant à la mesure réalisée sans avoir rien mis dans le faisceau (par exemple ref. [3] fig. 5).

Dans un premier temps, cette partie présente la méthode classique de traitement des données « petits angles » [4] qui est basée sur l'approximation $\cos (\theta) \simeq 1$. Dans certains cas (transmission de l'échantillon nettement inférieure à 1 , diffusion importante de l'environnement de l'échantillon), cette approximation doit être évitée. Le traitement des données est alors moins simple et requière des mesures complémentaires [5]. Cet aspect est exposé dans un second temps.

Convention de notation: lorsqu'une grandeur $G$ dépend de trois paramètres $a, b$ et $c$, nous la notons $G(a, b, c)$. Mais si $G$ dépend du rapport $a / b$ quel que soit le couple $(a, b)$, nous la notons $G(a / b, c)$ etc... Nous notons $\mathcal{R}$ l'ensemble de toutes les variables qui correspondent aux réglages du spectromètre (collimation, longueur d'onde, distance échantillon détecteur).

\subsection{Approximation « petits angles »}

Soit $J$ le flux du faisceau de neutrons qui éclaire l'aire $A$ de la surface d'un échantillon en ayant une incidence normale. Soit une couche de l'échantillon parallèle à cette surface, d'épaisseur élémentaire $d z$ et située à la distance $z$ de la surface (cf Fig. 5). Le flux incident est atténué d'un facteur $\exp \left(-\mu_{\mathrm{s}} z\right)$ par l'épaisseur de l'échantillon située entre 0 et $z$, où $\mu_{\mathrm{s}}$ est le coefficient d'atténuation linéique de l'échantillon. Dans une direction $\theta$ donnée et pour une unité d'angle solide, une fraction $\tilde{\sigma}_{\mathrm{s}}(\theta)$ de ce flux atténué est diffusée par l'élément de volume $A \times d r$. L'intensité diffusée par cette couche est ellemême atténuée par l'épaisseur $\left(Z_{\mathrm{s}}-z\right) / \cos (\theta)$ qu'il reste à parcourir dans la direction $\theta$, où $Z_{\mathrm{s}}$ est 


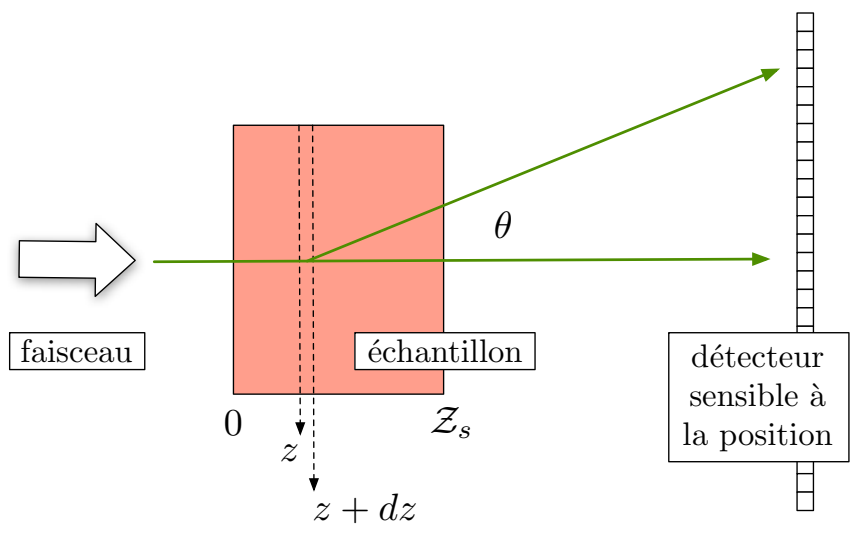

Figure 5. Diffusion par une couche élémentaire: le faisceau incident est atténué par l'épaisseur de l'échantillon située entre 0 et $z$, l'intensité diffusée est atténuée par celle située entre $z$ et $Z_{\mathrm{s}}$.

l'épaisseur totale. L'approximation classique " petits angles » consiste à supposer $\cos (\theta) \simeq 1$, ce facteur d'atténuation devenant $\exp \left(-\mu_{\mathrm{s}}\left(Z_{\mathrm{s}}-z\right)\right.$ ). L'intensité diffusée $I_{\mathrm{s}}$ (nombre de neutrons détectés par unité de temps) par l'ensemble des couches successives est donc donnée par l'intégrale :

$$
I_{\mathrm{s}}(\boldsymbol{q})=J A\left[\int_{0}^{Z_{\mathrm{s}}} d z \cdot e^{-\mu_{\mathrm{s}} z} \cdot \tilde{\sigma}_{\mathrm{s}}(\boldsymbol{q}) \cdot e^{-\mu_{\mathrm{s}}\left(Z_{\mathrm{s}}-z\right)}\right] \Delta \Omega \epsilon=J A \epsilon \Delta \Omega \times Z_{\mathrm{s}} e^{-\mu_{\mathrm{s}} Z_{\mathrm{s}}} \tilde{\sigma}_{\mathrm{s}}(\boldsymbol{q})
$$

où $\Delta \Omega$ est l'angle solide du détecteur vu depuis l'échantillon et $\epsilon$ son efficacité. Le facteur $\mathcal{T}_{\mathrm{s}}=$ $\exp \left(-\mu_{\mathrm{s}} Z_{\mathrm{s}}\right)$, appelé « transmission de l'échantillon », représente le facteur d'atténuation du faisceau transmis à travers l'échantillon. Il s'évalue au moyen d'un détecteur placé à angle nul en effectuant le rapport des intensités mesurées avec et sans échantillon. Le produit $J A \epsilon$ dépend uniquement de la configuration et des réglages du spectromètre. Nous noterons $\mathcal{R}$ l'ensemble des paramètres de cette configuration et supposerons que l'intensité est mesurée en fonction de la position $\boldsymbol{r}_{3}$ sur un multidétecteur. Ainsi :

$$
I_{\mathrm{s}}\left(\mathcal{R}, \boldsymbol{r}_{3}\right)=Z_{\mathrm{s}} \mathcal{T}_{\mathrm{s}} \times \underbrace{J A \epsilon \times \Delta \Omega \times \tilde{\sigma}_{\mathrm{s}}(\boldsymbol{q})}_{F_{\mathrm{s}}\left(\mathcal{R}, \boldsymbol{r}_{3}\right)}
$$

et nous appellerons $F_{\mathrm{s}}\left(\mathcal{R}, \boldsymbol{r}_{3}\right)$ la « fonction de diffusion » de l'échantillon. C'est cette grandeur que dans un premier temps il s'agit d'extraire des différentes mesures réalisées.

Remarque concernant l'Eq. 3.1 et la diffusion multiple : Dans cette expression, l'intensité diffusée par les atomes situés entre $z$ et $z+d z$ est atténuée par l'épaisseur de l'échantillon que les neutrons ont encore à parcourir avant d'en sortir. Cette atténuation est due à la somme de la section efficace d'absorption (les neutrons peuvent être absorbés par un noyau et produire une réaction nucléaire) et de la section efficace de diffusion. Si cette dernière est importante, l'Eq. 3.1 suppose donc qu'un neutron puisse être diffusé plusieurs fois. Ce qui est contradictoire avec l'hypothèse d'une diffusion unique (cf. 1.3). En fait pour un neutron, la section efficace de diffusion d'un atome est la somme d'une section efficace cohérente (proportionnelle à la densité spectrale de puissance de la densité) et d'une section efficace dite « incohérente » (cf. partie 6.1) qui est responsable d'une diffusion uniforme sur $4 \pi$ sr. Cette dernière étant déjà isotrope, elle n'est donc pas affectée par de multiple diffusions. Ainsi l'Eq. 3.1 prend en compte l'atténuation du faisceau diffusé par cette diffusion incohérente tout en restant dans le cadre d'une hypothèse de diffusion cohérente unique. 


\section{2 Échantillon nu}

Dans la pratique l'équation 3.2 n'est pas utilisable directement car au moins deux contributions s'ajoutent à la mesure du spectre de l'échantillon. La première est le bruit de fond $I_{\mathrm{BK}}$ qui est lié à la fois aux neutrons ne provenant pas directement du faisceau (fonction du réglage $\mathcal{R}$ ) et à l'électronique du détecteur (fonction du pixel du détecteur situé en $\boldsymbol{r}_{3}$ ). Ce bruit de fond est mesuré en plaçant un matériau absorbant les neutrons à la place de l'échantillon, généralement du carbure de bore doublé de cadmium. La seconde contribution $F_{\mathrm{b}}$ provient du faisceau qui ayant traversé l'échantillon et du fait de sa divergence, déborde sur les parties du détecteur réalisant les mesures aux angles les plus petits. Une mesure $I_{\mathrm{b}}$ sans échantillon donne :

$$
I_{\mathrm{b}}\left(\mathcal{R}, \boldsymbol{r}_{3}\right)=F_{\mathrm{b}}(\mathcal{R})+I_{\mathrm{BK}}\left(\mathcal{R}, \boldsymbol{r}_{3}\right)
$$

En présence d'un échantillon, la contribution $F_{\mathrm{b}}(\mathcal{R})$ du faisceau traversant l'échantillon sans avoir été diffusé est atténuée par la transmission de l'échantillon. L'Eq. 3.2 est remplacée par :

$$
I_{\mathrm{s}}\left(\mathcal{R}, \boldsymbol{r}_{3}\right)=\mathcal{T}_{\mathrm{s}}\left(Z_{\mathrm{s}} F_{\mathrm{s}}\left(\mathcal{R}, \boldsymbol{r}_{3}\right)+F_{\mathrm{b}}(\mathcal{R})\right)+I_{\mathrm{BK}}\left(\mathcal{R}, \boldsymbol{r}_{3}\right)
$$

La fonction de diffusion de l'échantillon peut ainsi être extraite de ces quatre mesures réalisées pour un même réglage du spectromètre: $I_{\mathrm{s}}, \mathcal{T}_{\mathrm{s}}, I_{\mathrm{b}}$ et $I_{\mathrm{BK}}$. Les relations 3.3 et 3.4 donnent :

$$
F_{\mathrm{s}}\left(\mathcal{R}, \boldsymbol{r}_{3}\right)=\frac{I_{\mathrm{s}}\left(\mathcal{R}, \boldsymbol{r}_{3}\right)-I_{\mathrm{BK}}\left(\mathcal{R}, \boldsymbol{r}_{3}\right)}{Z_{\mathrm{s}} \mathcal{T}_{\mathrm{s}}}-\frac{I_{\mathrm{b}}\left(\mathcal{R}, \boldsymbol{r}_{3}\right)-I_{\mathrm{BK}}\left(\mathcal{R}, \boldsymbol{r}_{3}\right)}{Z_{\mathrm{s}}}
$$

\section{3 Échantillon dans un contenant}

L'échantillon est souvent placé dans un environnement particulier qui peut être très simple, comme le récipient contenant un liquide, ou plus important, comme un rhéomètre de Couette ou une cellule haute pression. Dans tous les cas, les faces de ce contenant placées de part et d'autre de l'échantillon par rapport à la direction du faisceau participent à la diffusion mesurée (Fig. 6). Pour en extraire la contribution de l'échantillon seul, il est nécessaire de mesurer l'intensité diffusée $I_{\mathrm{EC}}\left(\mathcal{R}, \boldsymbol{r}_{3}\right)$ et la transmission $\mathcal{T}_{\text {EC }}$ de ce contenant vide de tout échantillon. Dans ce cas on a :

$$
I_{\mathrm{EC}}\left(\mathcal{R}, \boldsymbol{r}_{3}\right)=\mathcal{T}_{\mathrm{EC}}\left(Z_{\mathrm{EC}} F_{\mathrm{EC}}\left(\mathcal{R}, \boldsymbol{r}_{3}\right)+F_{\mathrm{b}}(\mathcal{R})\right)+I_{\mathrm{BK}}\left(\mathcal{R}, \boldsymbol{r}_{3}\right)
$$

Notons $I_{\mathrm{S}}\left(\mathcal{R}, \boldsymbol{r}_{3}\right)$ et $\mathcal{T}_{\mathrm{S}}$ l'intensité diffusée et la transmission de l'échantillon dans ce contenant. Les hétérogénéités responsables de la diffusion de l'échantillon seul (notée par l'indice $\left.\right|_{\mathrm{s}}$ ) et celles du contenant vide (indice $\left.\right|_{\mathrm{EC}}$ ) n'ayant aucune corrélations, leurs contributions s'ajoutent à l'intensité totale (indice $\left.\right|_{S}$ ). Soit :

$$
I_{\mathrm{S}}\left(\mathcal{R}, \boldsymbol{r}_{3}\right)=\mathcal{T}_{S}\left(Z_{\mathrm{s}} F_{S}\left(\mathcal{R}, \boldsymbol{r}_{3}\right)+Z_{\mathrm{EC}} F_{\mathrm{EC}}\left(\mathcal{R}, \boldsymbol{r}_{3}\right)+F_{\mathrm{b}}(\mathcal{R})\right)+I_{\mathrm{BK}}\left(\mathcal{R}, \boldsymbol{r}_{3}\right)
$$

Les équations 3.6 et 3.7 donnent :

$$
F_{\mathrm{s}}\left(\mathcal{R}, \boldsymbol{r}_{3}\right)=\frac{I_{\mathrm{S}}\left(\mathcal{R}, \boldsymbol{r}_{3}\right)-I_{\mathrm{BK}}\left(\mathcal{R}, \boldsymbol{r}_{3}\right)}{Z_{\mathrm{s}} \mathcal{T}_{\mathrm{S}}}-\frac{I_{\mathrm{EC}}\left(\mathcal{R}, \boldsymbol{r}_{3}\right)-I_{\mathrm{BK}}\left(\mathcal{R}, \boldsymbol{r}_{3}\right)}{Z_{\mathrm{s}} \mathcal{T}_{\mathrm{EC}}} .
$$

La mesure du contenant seul prend en compte également la contribution $F_{\mathrm{b}}(\mathcal{R})$ du faisceau transmis à travers l'échantillon qu'il n'est donc plus utile de mesurer séparément. Les mesures nécessaires sont maintenant $I_{\mathrm{S}}, \mathcal{T}_{\mathrm{S}}, I_{\mathrm{EC}}, \mathcal{T}_{\mathrm{EC}}$ et $I_{\mathrm{BK}}$ réalisées pour un même réglage du spectromètre. 


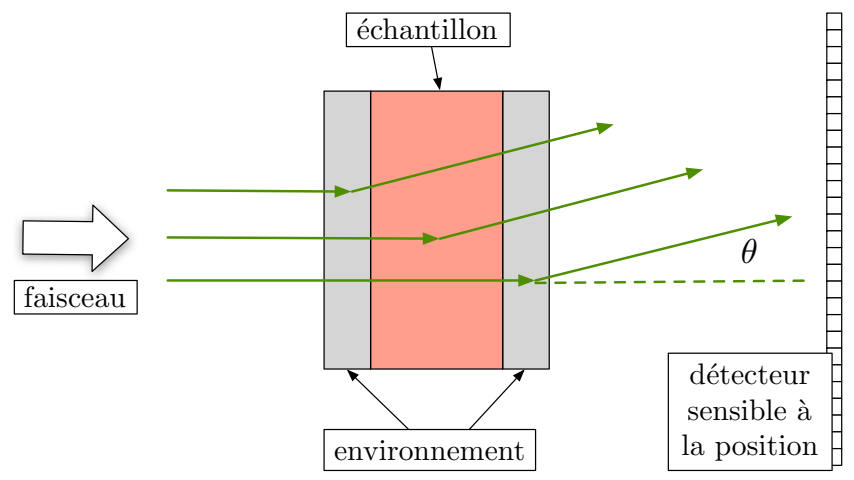

Figure 6. Diffusion d'un échantillon placé entre deux faces de même épaisseur d'un contenant.

\subsection{Correction « grands angles »}

L'épaisseur de l'échantillon traversée par les neutrons diffusés dépend de l'angle (cf. Fig. 6). Les équations 3.1 et 3.2 doivent être remplacées [5] par

$I_{\mathrm{S}}(\boldsymbol{q}, \theta)=J A\left[\int_{0}^{Z_{\mathrm{S}}} d z \cdot e^{-\mu_{\mathrm{S}} z} \cdot \tilde{\sigma}_{\mathrm{s}}(q) \cdot e^{-\mu_{\mathrm{S}}\left(Z_{\mathrm{s}}-z\right) / \cos \theta}\right] \Omega \epsilon=\left[\int_{0}^{Z_{\mathrm{S}}} d z \cdot e^{-\mu_{\mathrm{S}}\left(z+\left(Z_{\mathrm{s}}-z\right) / \cos \theta\right)}\right] F_{\mathrm{S}}(\boldsymbol{q})$

que l'on peut réécrire sous la forme :

$$
\begin{array}{r}
I_{\mathrm{s}}(\mathcal{R}, \theta, \boldsymbol{q})=Z_{\mathrm{s}} \mathcal{T}_{\mathrm{s}}(\theta) \times F_{\mathrm{s}}(\mathcal{R}, \boldsymbol{q}) \\
\text { avec } \quad \mathcal{T}_{\mathrm{s}}(\theta)=\frac{\mathcal{T}_{\mathrm{s}}\left(1-\mathcal{T}_{\mathrm{s}}{ }^{(\theta)}\right)}{-a(\theta) \ln \left(\mathcal{T}_{\mathrm{s}}\right)} \quad \text { et } \quad a(\theta)=\frac{1}{\cos (\theta)}-1 .
\end{array}
$$

La grandeur $\mathcal{T}(\theta)$ représente la dépendance angulaire de la transmission. Son comportement apparaît plus clairement en utilisant un développement en série de Taylor :

$$
\begin{array}{r}
\mathcal{T}_{\mathrm{s}}(\theta)=\mathcal{T}_{\mathrm{s}} \times \mathcal{E}_{1}\left(a(\theta) \ln \left(\mathcal{T}_{\mathrm{s}}\right)\right) \\
\text { avec } \mathcal{E}_{1}(x)=1+x / 2+x^{2} / 6+x^{3} / 24+x^{4} / 120+\cdots
\end{array}
$$

Cette expression montre que l'approximation « petits angles » $\cos (\theta) \simeq 1$ ne doit pas être estimée au regard de la valeur de $\theta$, mais plutôt à celle de $a(\theta) \ln \left(\mathcal{T}_{\mathrm{s}}\right)$. En particulier cette approximation n'est plus acceptable à grand vecteur de diffusion et lorsque la transmission est nettement inférieure à 1 (cf. Fig. 7). Par exemple, une couche de $1 \mathrm{~mm}$ d'eau a une transmission à angle nul de l'ordre de 0.5. Dans ce cas des calculs plus rigoureux sont nécessaires pour garantir une normalisation correcte des spectres (cf. partie 4), l'étalonnage du spectromètre (cf. partie 5) et la soustraction du bruit de fond inhérent à l'échantillon (cf. partie 6). On peut montrer que dans le cas où l'échantillon est placé dans un environnement symétrique de part et d'autre de l'échantillon, l’Eq. 3.8 devient :

$$
\begin{aligned}
F_{\mathrm{S}}\left(\mathcal{R}, \boldsymbol{r}_{3}\right)=\frac{I_{\mathrm{S}}\left(\mathcal{R}, \boldsymbol{r}_{3}\right)-I_{\mathrm{BK}}\left(\mathcal{R}, \boldsymbol{r}_{3}\right)}{Z_{\mathrm{S}} \mathcal{T}_{\mathrm{S}} \alpha_{\mathrm{S}}(\theta)} & -\delta_{\mathrm{S}}(\theta)\left(\frac{I_{\mathrm{EC}}\left(\mathcal{R}, \boldsymbol{r}_{3}\right)-I_{\mathrm{BK}}\left(\mathcal{R}, \boldsymbol{r}_{3}\right)}{\beta_{\mathrm{EC}}(\theta) Z_{\mathrm{S}} \mathcal{T}_{\mathrm{EC}}}\right) \\
& +\frac{\delta_{\mathrm{S}}(\theta)}{Z_{\mathrm{S}}}\left(\frac{\left.\mathcal{T}_{\mathrm{EC}}^{a(\theta)}-\frac{\mathcal{T}_{\mathrm{S}}^{a(\theta)}}{\beta_{\mathrm{EC}}(\theta)}\right) F_{\mathrm{b}}(\theta)}{\beta_{\mathrm{S}}(\theta)} \boldsymbol{r}_{3}\right) .
\end{aligned}
$$




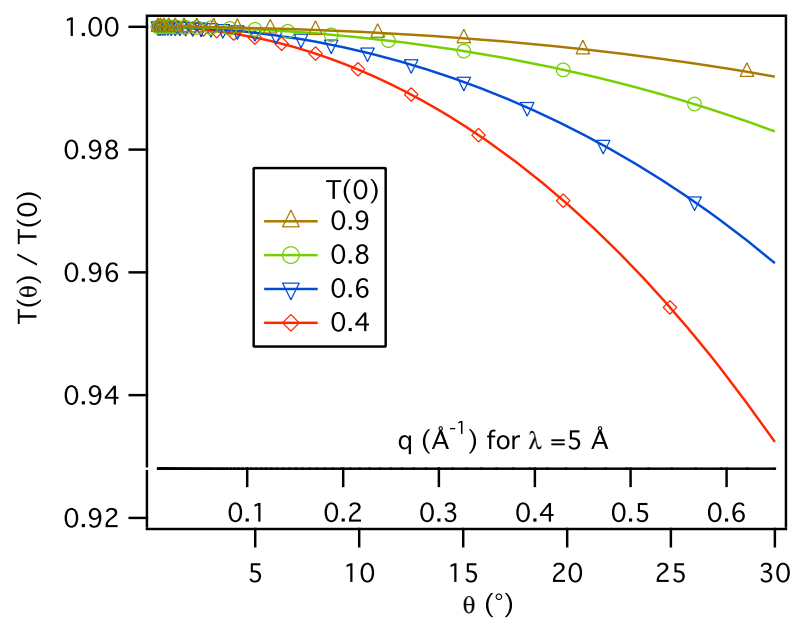

Figure 7. Variation angulaire relative $\mathcal{T}_{\mathrm{s}}(\theta) / \mathcal{T}_{\mathrm{s}}$ de la transmission (Eq. 3.10) calculée pour différentes valeurs de la transmission $\mathcal{T}_{\mathrm{s}}$ mesurée à angle nul.

Dans cette expression les parties en rouge sont les corrections apportées par rapport à 1'Eq. 3.8. Ces ajouts font intervenir les fonctions angulaires $\alpha_{\mathrm{S}}(\theta), \beta_{\mathrm{S}}(\theta)$ et $\delta_{\mathrm{S}}(\theta)$ :

$$
\begin{aligned}
& \alpha_{\mathrm{S}}(\theta)=\mathcal{E}_{2}\left(a(\theta) \ln \left(\mathcal{T}_{\mathrm{EC}}\right)\right) \times \mathcal{E}_{1}\left(a(\theta) \ln \left(\mathcal{T}_{\mathrm{S}} / \mathcal{T}_{\mathrm{EC}}\right)\right) \\
& \beta_{\mathrm{S}}(\theta)=\mathcal{E}_{3}\left(a(\theta) \ln \left(\mathcal{T}_{\mathrm{S}} / \mathcal{T}_{\mathrm{EC}}^{1 / 2}\right)\right) \times \mathcal{E}_{4}\left(a(\theta) \ln \left(\mathcal{T}_{\mathrm{EC}}\right)\right) \\
& \delta_{\mathrm{S}}(\theta)=\frac{\beta_{\mathrm{S}}(\theta)}{\alpha_{\mathrm{S}}(\theta)} \simeq 1
\end{aligned}
$$

avec $\mathcal{E}_{2}(x)=1+x / 2+x^{2} / 8+x^{3} / 48+x^{4} / 384+\cdots, \quad \mathcal{E}_{3}(x)=1+x / 2+x^{2} / 4+x^{3} / 12+x^{4} / 48$ $+\cdots$ et $\mathcal{E}_{4}(x)=1+x / 4+x^{2} / 24+x^{3} / 192+x^{4} / 1920+\cdots$. Aux quatre mesures précédemment nécessaires $\left(I_{\mathrm{BK}}, I_{\mathrm{EC}}, \mathcal{T}_{\mathrm{S}}\right.$ et $I_{\mathrm{S}}$, cf. Eq. 3.8) s'ajoute celle du faisceau seul $I_{\mathrm{b}}$ (donnée par l'Eq. 3.3) dont la contribution n'est pas entièrement prise en compte par la mesure de l'intensité diffusée par le contenant vide.

\section{NORMALISATION DES SPECTRES}

\subsection{Diffuseur de référence}

Les étapes précédentes de soustraction du bruit de fond, de la contribution du faisceau transmis et de celle de l'environnement de l'échantillon, conduisent à la fonction de diffusion $F_{\mathrm{s}}\left(\mathcal{R}, \boldsymbol{r}_{3}\right)=$ $J A \epsilon \times \Delta \Omega \times \tilde{\sigma}_{\mathrm{s}}(\boldsymbol{q})$ de l'échantillon. Les spectromètres sont équipés d'un détecteur sensible à la position (multidétecteur) pour l'acquisition simultanée des données en fonction de la position $\boldsymbol{r}_{3}(\theta, \varphi)$ de ses pixels à laquelle correspond un vecteur $\boldsymbol{q}$. En général, ce multidétecteur est plan (et non sphérique) et perpendiculaire au faisceau (Fig. 1). En toute rigueur, cela conduit à une dépendance angulaire de l'angle solide $\Delta \Omega$ et de l'efficacité $\epsilon$ de chaque pixel.

Chaque pixel d'aire $s$ est à une distance $L_{2} / \cos (\theta)$ de l'échantillon et l'aire de sa projection sur une sphère centrée sur l'échantillon est $s \times \cos (\theta)$. Son angle solide vu depuis l'échantillon s'écrit donc :

$$
\Delta \Omega(\theta)=\frac{s \times \cos (\theta)}{\left(L_{2} / \cos (\theta)\right)^{2}}=\frac{s}{L_{2}^{2}} \cos (\theta)^{3} .
$$


De façon similaire, l'épaisseur de gaz du détecteur traversée par un neutron augmente $\operatorname{comme} 1 / \cos (\theta)$, d'où une fonction angulaire de l'efficacité de détection qui s'écrit :

$$
\epsilon(\lambda, \theta)=1-e^{-\mu(\lambda) z_{0} / \cos (\theta)} .
$$

Equation qui remplace l'Eq. 2.14. Afin de dissocier l'efficacité à angle nul de sa dépendance angulaire, nous écrirons par la suite: $\epsilon(\lambda, \theta)=\epsilon(\lambda, 0) \epsilon_{r}(\lambda, \theta)$. De plus, chaque pixel du multidétecteur possède en général ses propres seuils de discrimination du signal, c'est à dire sa propre efficacité. Essentiellement cela revient à introduire dans l'efficacité un facteur $\epsilon_{p}\left(\boldsymbol{r}_{3}\right)$ qui dépend de la position $\boldsymbol{r}_{3}$ du pixel et dont la moyenne est 1 . Finalement on peut écrire :

$$
\epsilon\left(\lambda, \theta, \boldsymbol{r}_{3}\right)=\epsilon(\lambda, 0) \epsilon_{r}(\lambda, \theta) \epsilon_{p}\left(\boldsymbol{r}_{3}\right)
$$

La fonction de diffusion mesurée s'écrit donc :

$$
F_{\mathrm{S}}\left(\mathcal{R}, \boldsymbol{r}_{3}\right)=J A \epsilon(\lambda, 0) \times \epsilon_{r}(\lambda, \theta) \Delta \Omega(\theta) \times \epsilon_{p}\left(\boldsymbol{r}_{3}\right) \times \tilde{\sigma}_{\mathrm{s}}(\boldsymbol{q}) .
$$

Usuellement, l'expérimentateur s'affranchit du facteur $J A \epsilon(\lambda, 0) \times \epsilon_{r}(\lambda, \theta) \Delta \Omega(\theta) \times \epsilon_{p}\left(\boldsymbol{r}_{3}\right)$ en mesurant un échantillon dit « de référence », dont la diffusion est uniforme sur $4 \pi$ sr. C'est typiquement un échantillon dont la section efficace de diffusion est principalement incohérente (cf. partie 6.1) comme l'eau. Sa fonction de diffusion s'écrit: $F_{\text {ref }}\left(\lambda, \theta, \boldsymbol{r}_{3}\right)=J A \epsilon(\lambda, 0) \times \epsilon_{r}(\lambda, \theta) \Delta \Omega(\theta) \times \epsilon_{p}\left(\boldsymbol{r}_{3}\right) \times$ $\tilde{\sigma}_{\text {ref }}$, où $\tilde{\sigma}_{\text {ref }}$ est la section efficace différentielle par unité de volume de la référence et est indépendant de $\boldsymbol{q}$. Pour un réglage donné du spectromètre, le flux $J$, l'aire $A$ éclairée, l'efficacité des pixels du multidétecteur $\epsilon\left(\lambda, \theta, \boldsymbol{r}_{3}\right)$ et la dépendance angulaire de l'angle solide $\Delta \Omega(\theta)$ sont fixes et le rapport de la fonction de diffusion de l'échantillon à celle de la référence donne :

$$
\tilde{\sigma}_{\mathrm{s}}(\boldsymbol{q})=\frac{F_{\mathrm{s}}\left(\mathcal{R}, \boldsymbol{r}_{3}\right)}{F_{\text {ref }}\left(\mathcal{R}, \boldsymbol{r}_{3}\right)} \tilde{\sigma}_{\text {ref }}
$$

Ce qui revient à exprimer $\tilde{\sigma}_{\mathrm{s}}(\boldsymbol{q})$ dans une unité relative à l'échantillon de référence.

\subsection{Gagner du temps et de la précision}

Dans l'équation 4.5, les fonctions de diffusion de l'échantillon et de la référence sont supposées être mesurées dans la même configuration $\mathcal{R}$ du spectromètre. Toutefois, la référence diffusant de façon uniforme sur $4 \pi \mathrm{sr}$, son intensité diffusée mesurée dans une configuration « petits angles » $\mathcal{R}_{\mathrm{PA}}$ est trop faible pour permettre une statistique satisfaisante (l'intensité diffusée de la référence varie comme le flux c'est à dire $\theta^{4}$ cf. Eq. 2.11). Ainsi en effectuant le rapport des fonctions de diffusion de l'Eq. 4.5, l'erreur statistique sur $\tilde{\sigma}_{\mathrm{s}}(\boldsymbol{q})$ risque d'être très supérieure à celle de $F_{\mathrm{s}}\left(\mathcal{R}_{\mathrm{PA}}, \boldsymbol{r}_{3}\right)$. Pour maintenir ces erreurs à des niveaux comparables, il est conseillé :

- de mesurer $F_{\text {ref }}\left(\mathcal{R}_{\mathrm{PA}}, \boldsymbol{r}_{3}\right)$ le temps nécessaire pour que sa valeur moyenne sur toutes les positions $\boldsymbol{r}_{3}$ du multidétecteur, $\left\langle F_{\text {ref }}\left(\mathcal{R}_{\mathrm{PA}}, \boldsymbol{r}_{3}\right)\right\rangle_{\boldsymbol{r}_{3}}$, soit connue avec la précision souhaitée. L'erreur statistique sur cette moyenne est diminuée d'un facteur égal à la racine carrée du nombre de positions.

- de mesurer $F_{\text {ref }}\left(\mathcal{R}_{\mathrm{GA}}, \boldsymbol{r}_{3}\right)$ dans une configuration $\mathcal{R}_{\mathrm{GA}}$ " grands angles » pour laquelle le flux est important.

- puis de calculer :

$$
\tilde{\sigma}_{\mathrm{s}}(\boldsymbol{q})=\frac{F_{\mathrm{s}}\left(\mathcal{R}_{\mathrm{PA}}, \boldsymbol{r}_{3}\right)}{F_{\mathrm{ref}}\left(\mathcal{R}_{\mathrm{GA}}, \boldsymbol{r}_{3}\right)} \times \frac{\left\langle F_{\mathrm{ref}}\left(\mathcal{R}_{\mathrm{GA}}, \boldsymbol{r}_{3}\right)\right\rangle_{\boldsymbol{r}_{3}}}{\left\langle F_{\mathrm{ref}}\left(\mathcal{R}_{\mathrm{PA}}, \boldsymbol{r}_{3}\right)\right\rangle_{\boldsymbol{r}_{3}}} \times \tilde{\sigma}_{\mathrm{ref}}
$$

Remarque importante : Dans l'équation 4.5, les fonctions angulaires $\Omega(\theta)$ et $\epsilon_{r}(\lambda, \theta)$ disparaissent car elles interviennent en préfacteur de deux expressions dont on fait le rapport. Par contre, l'utilisation de l'expression 4.6 suppose que ces dépendances angulaires (corrections « grands angles ») ont bien été prises en compte dans le calcul des fonctions de diffusion car elles sont différentes dans les deux configurations. 


\subsection{Importance des corrections « grands angles »}

Dans l'équation 4.5, la fonction de diffusion de l'échantillon est divisée par celle de la référence. Très souvent cette référence présente une transmission assez faible (par exemple la transmission d'une épaisseur d'1 mm d'eau est de l'ordre de 0.5) tandis que celle de l'échantillon est en général beaucoup plus proche de 1. Lorsque les fonctions de diffusion sont calculées de façon classique en faisant l'approximation « petits angles » (Eq. 3.8), la normalisation d'un spectre (Eq. 4.5) introduit une déformation angulaire qui est approximativement proportionnelle au rapport $\mathcal{T}_{\text {ref }}(\theta) / \mathcal{T}_{\mathrm{s}}(\theta)$. Cette déformation peut être importante notamment aux grands angles (cf. Fig. 7) et peut poser des problèmes en particulier lorsque cette partie des spectres est utilisée pour évaluer le bruit de fond inhérent à l'échantillon (cf. partie 6). Les corrections dites « grands angles » conduisant à l'équation 3.12 sont alors toujours préférables.

\section{5. ÉTALONNAGE DU SPECTROMÈTRE ET MESURES ABSOLUES}

\subsection{Quel est le problème?}

La normalisation du spectre d'un échantillon fournit sa section efficace différentielle de diffusion exprimée en une unité, $\tilde{\sigma}_{\text {ref }}$, relative à la référence utilisée. En principe, si la section efficace d'absorption est négligeable devant la section efficace totale de diffusion (ce qui est le cas de l'eau), alors les neutrons qui ne sont pas transmis à travers la référence sont diffusés dans une direction quelconque. On peut écrire $\int_{0}^{4 \pi}\left(Z_{\text {ref }} \mathcal{T}_{\text {ref }} \tilde{\sigma}_{\text {ref }}\right) d \Omega=1-\mathcal{T}_{\text {ref. }}$ Si $\tilde{\sigma}_{\text {ref }}$ est uniforme sur $4 \pi$ sr (c'est à dire isotrope), on obtient :

$$
\tilde{\sigma}_{\text {ref }}=\frac{1-\mathcal{T}_{\text {ref }}}{Z_{\text {ref }} \mathcal{T}_{\text {ref }} 4 \pi}
$$

Toutefois dans la pratique $\tilde{\sigma}_{\text {ref }}$ n'est malheureusement pas isotrope. En toute rigueur et dans le cas de l'eau par exemple, deux effets concourent à sa déformation [6].

1. la diffusion inélastique est très importante dans le cas de l'eau car la masse d'un proton est pratiquement identique à celle d'un neutron. Cela a deux conséquences [7] :

- la section efficace totale de l'eau (intégrée sur l'énergie des neutrons diffusés et sur $4 \pi$ sr) augmente avec la longueur d'onde incidente et donc la transmission diminue;

- la section efficace différentielle décroît avec l'angle.

A cela s'ajoute une efficacité de détection qui dépend de la longueur d'onde (Eq. 4.2) et qui modifie la mesure (Eq. 1.17). Toutefois l'anisotropie qui résulte de la diffusion inélastique est négligeable pour des neutrons froids d'une énergie de l'ordre de $1 \mathrm{meV}$ (cf. par exemple ref. [8]).

2. la diffusion multiple et la forme particulière du récipient contenant la référence concourent à une anisotropie très importante de la diffusion. En effet, si l'épaisseur de ce contenant est suffisamment faible pour éviter la diffusion multiple dans les directions presque parallèles au faisceau, ce n'est pas le cas dans les directions presque perpendiculaires. Du fait de l'épaisseur traversée dans ces directions, la probabilité d'être diffusé de nombreuse fois est très grande et pratiquement aucun neutron ne sort de l'échantillon dans ces directions. Par conservation du flux, on en déduit un excès d'intensité diffusée dans les directions où l'épaisseur est plus faible (vers l'avant et l'arrière).

Cette anisotropie de la diffusion de l'eau est sans effet sur le profil du spectre mesuré aux petits angles (autrement dit, le spectre reste plat et pratiquement indépendant de $q$ ), mais elle est suffisante pour modifier son niveau moyen. La section efficace différentielle apparente $\tilde{\sigma}_{\text {ref }}$ qui en est déduite est :

- supérieure à la valeur moyenne sur $4 \pi$ sr donnée par l'équation 5.1

- dépend du couple $\left(\lambda, L_{2}\right)$ choisi pour faire les mesures.

D'où la nécessité de mesurer la valeur effective de $\tilde{\sigma}_{\text {ref }}$ correspondant à la géométrie et aux réglages choisis. 


\subsection{Comment faire des mesures en unité absolue ?}

Les équations 4.4 et 4.5 (ou 4.6) permettent d'écrire :

$$
\tilde{\sigma}_{\text {ref }}=\left\langle\frac{F_{\text {ref }}\left(\mathcal{R}, \boldsymbol{r}_{3}\right)}{\epsilon_{r}(\lambda, \theta) \Delta \Omega(\theta)}\right\rangle_{r_{3}} \times \frac{1}{J A \epsilon(\lambda, 0)} .
$$

Dans cette expression, le facteur de gauche correspond à la fonction de diffusion de la référence corrigée de toutes les dépendances angulaires. Le rapport $F_{\text {ref }}\left(\mathcal{R}, \boldsymbol{r}_{3}\right) / \epsilon_{r}(\lambda, \theta) \Delta \Omega(\theta)$ ne présente donc pas de déviation systématique et on en fait la moyenne sur tout le détecteur.

La quantité $J A \epsilon(\lambda, 0)$ correspond à la mesure de l'intensité du faisceau ayant une incidence directe sur le détecteur. Pour éviter de le saturer, cette mesure est réalisée avec un atténuateur. Si la transmission de cet atténuateur n'est pas connue, elle est mesurée en utilisant un diffuseur tel qu'il répartisse l'intensité du faisceau sur tout le détecteur, évitant ainsi une saturation (typiquement un morceau de graphite). Le rapport des intensités totales enrégistrées avec et sans graphite fournit la transmission recherchée. Cette méthode est la plus rapide et la plus efficace pour exprimer $\tilde{\sigma}_{\text {ref }}$ en unité absolue $\left(\mathrm{cm}^{-1}\right)$.

\section{3 À quoi sert l'étalonnage?}

La section efficace différentielle de diffusion $\tilde{\sigma}_{\mathrm{s}}$ est la transformée de Fourier de la fonction d'autocorrélation des fluctuations la densité de longueur de diffusion. Ses variations en fonction de $\boldsymbol{q}$ donnent donc une information sur les longueurs caractéristiques de l'échantillon. En exprimant $\tilde{\sigma}_{\mathrm{s}}$ en unité absolue, on accède de plus à une information quantitative sur l'amplitude des fluctuations de cette densité qui permet par exemple de déterminer la masse moléculaire d'objets en solution ou la surface spécifique d'un matériaux poreux.

Par ailleurs, il est important de réaliser que la section efficace apparente de la référence dépend de la configuration du spectromètre. Par exemple sur le spectromètre PACE (LLB), la section efficace différentielle apparente par unité de volume de l'eau varie entre 0.8 et $1.2 \mathrm{~cm}^{-1}$ selon que la mesure est réalisée dans une configuration « grands angles » ou « petits angles ». Dans l'équation 4.5, l'unité dans laquelle est exprimée $\tilde{\sigma}_{\mathrm{s}}$ dépend donc de la configuration. Concrètement, cela signifie que deux spectres du même échantillon obtenus dans deux domaines de vecteur de diffusion différents ne se raccorderont pas correctement à moins qu'ils ne soient exprimés en unité absolue.

\section{ASPECTS RELATIFS AUX ÉCHANTILLONS}

\subsection{Diffusion incohérente d'un neutron par les atomes}

La diffusion d'un neutron a ceci de particulier qu'elle est due à une interaction nucléaire. Cela implique que la longueur de diffusion d'un élément dépend de l'isotope. De plus si cet isotope possède un spin nucléaire $s$, le neutron ayant lui-même un spin 1/2, deux spins résultants sont possibles $s-1 / 2$ et $s+1 / 2$. A chacune de ces résultantes correspond une longueur de diffusion particulière notée respectivement $b_{-}$et $b_{+}$. En conséquence, même pour un milieu de densité homogène constitué d'un seul élément, une hétérogénéité de la densité de longueur de diffusion subsiste dans la plupart des cas.

Soit un milieu constitué de $\mathcal{M}$ atomes quelconques, $b_{\mathrm{m}}$ la longueur de diffusion de l'atome $m$ et $\rho_{\mathrm{m}}(\boldsymbol{r})$ la densité de probabilité de trouver cet atome en un lieu $\boldsymbol{r}$ :

$$
\rho_{\mathrm{m}}(\boldsymbol{r})=\delta\left(\boldsymbol{r}_{\mathrm{m}}-\boldsymbol{r}\right)
$$


où $\boldsymbol{r}_{\mathrm{m}}$ est la position de l'atome $m$. La densité de longueur de diffusion en ce lieu est la somme des densités individuelles :

$$
\tilde{b}(\boldsymbol{r})=\sum_{m=1}^{\mathcal{M}} b_{\mathrm{m}} \rho_{\mathrm{m}}(\boldsymbol{r})
$$

D’où la fonction de corrélation :

$$
\langle\tilde{b}(0) \tilde{b}(\boldsymbol{r})\rangle=\left\langle\sum_{\mathrm{m}} b_{\mathrm{m}} \rho_{\mathrm{m}}(0) \times \sum_{\mathrm{m}^{\prime}} b_{\mathrm{m}^{\prime}} \rho_{\mathrm{m}^{\prime}}(\boldsymbol{r})\right\rangle=\sum_{\mathrm{m}} \sum_{\mathrm{m}^{\prime}} b_{\mathrm{m}} b_{\mathrm{m}^{\prime}}\left\langle\rho_{\mathrm{m}}(0) \rho_{\mathrm{m}^{\prime}}(\boldsymbol{r})\right\rangle .
$$

Selon son isotope ou son spin résultant avec le neutron, chaque atome $m$ présente une longueur de diffusion particulière $b_{\mathrm{m}}$ parmi une distribution discrète de valeurs possibles dont la moyenne est $\bar{b}_{\mathrm{m}}$. En raison de l'argument du paragraphe 2.3, la fonction de corrélation moyenne qui est sondée s'écrit :

$$
\overline{\langle\tilde{b}(0) \tilde{b}(\boldsymbol{r})\rangle}=\overline{\sum_{\mathrm{m}} \sum_{\mathrm{m}^{\prime}} b_{\mathrm{m}} b_{\mathrm{m}}{ }^{\prime}\left\langle\rho_{\mathrm{m}}(0) \rho_{\mathrm{m}}{ }^{\prime}(\boldsymbol{r})\right\rangle}=\sum_{\mathrm{m}} \sum_{\mathrm{m}^{\prime}} \overline{b_{\mathrm{m}} b_{\mathrm{m}}{ }^{\prime}}\left\langle\rho_{\mathrm{m}}(0) \rho_{\mathrm{m}^{\prime}}(\boldsymbol{r})\right\rangle .
$$

En notant $b_{\mathrm{m}}=\bar{b}_{\mathrm{m}}+\Delta b_{\mathrm{m}}$, de façon similaire à ce que l'on a écrit pour l'Eq. 1.21, on obtient :

$$
\overline{b_{\mathrm{m}} b_{\mathrm{m}}}=\bar{b}_{\mathrm{m}} \bar{b}_{\mathrm{m}}+\overline{\Delta b_{\mathrm{m}} \Delta b_{\mathrm{m}}} .
$$

d'où :

$$
\overline{\langle\tilde{b}(0) \tilde{b}(\boldsymbol{r})\rangle}=\sum_{\mathrm{m}} \sum_{\mathrm{m}^{\prime}} \bar{b}_{\mathrm{m}} \bar{b}_{\mathrm{m}},\left\langle\rho_{\mathrm{m}}(0) \rho_{\mathrm{m}^{\prime}}(\boldsymbol{r})\right\rangle+\sum_{\mathrm{m}} \sum_{\mathrm{m}^{\prime}} \overline{\Delta b_{\mathrm{m}} \Delta b_{\mathrm{m}^{\prime}}}\left\langle\rho_{\mathrm{m}}(0) \rho_{\mathrm{m}}(\boldsymbol{r})\right\rangle .
$$

Le premier terme de cette somme contient une information sur la structure de l'échantillon. La moyenne $\bar{b}_{\mathrm{m}}$ est appelée « longueur de diffusion cohérente » de l'atome $m$ et son carré $\bar{b}_{\mathrm{m}}^{2}$ « section efficace différentielle de diffusion cohérente » de $m$. De la même façon que pour l'Eq. 6.2, la densité locale de longueur de diffusion cohérente est définie par :

$$
\tilde{b}_{\mathrm{c}}(\boldsymbol{r})=\sum_{m=1}^{\mathcal{M}} \bar{b}_{\mathrm{m}} \rho_{\mathrm{m}}(\boldsymbol{r})
$$

d'où

$$
\sum_{\mathrm{m}} \sum_{\mathrm{m}^{\prime}} \bar{b}_{\mathrm{m}} \bar{b}_{\mathrm{m}},\left\langle\rho_{\mathrm{m}}(0) \rho_{\mathrm{m}},(\boldsymbol{r})\right\rangle=\left\langle\sum_{\mathrm{m}} \bar{b}_{\mathrm{m}} \rho_{\mathrm{m}}(0) \sum_{\mathrm{m}^{\prime}} \bar{b}_{\mathrm{m}} \rho_{\mathrm{m}},(\boldsymbol{r})\right\rangle=\left\langle\tilde{b}_{\mathrm{c}}(0) \tilde{b}_{\mathrm{c}}(\boldsymbol{r})\right\rangle .
$$

Concernant le deuxième terme de l'Eq. 6.6, pour deux atomes particuliers $m$ et $m^{\prime}$, la fonction de corrélation de la densité s'écrit :

$$
\begin{aligned}
\left\langle\rho_{\mathrm{m}}(0) \rho_{\mathrm{m}},(\boldsymbol{r})\right\rangle= & \left\langle\delta\left(\boldsymbol{r}_{\mathrm{m}}\right) \delta\left(\boldsymbol{r}_{\mathrm{m}},-\boldsymbol{r}\right)\right\rangle \\
= & \frac{1}{V} \int_{V} \delta\left(\boldsymbol{R}+\boldsymbol{r}_{\mathrm{m}}\right) \delta\left(\boldsymbol{R}+\boldsymbol{r}_{\mathrm{m}},-\boldsymbol{r}\right) d^{3} \boldsymbol{R} \\
& =\frac{1}{V} \delta\left(\left(\boldsymbol{r}_{\mathrm{m}},-\boldsymbol{r}_{\mathrm{m}}\right)-\boldsymbol{r}\right) .
\end{aligned}
$$

Or pour deux atomes différents, les valeurs $b_{\mathrm{m}}$ et $b_{\mathrm{m}}$ ' des longueurs de diffusion qu'ils adoptent parmi les possibilités qui leurs sont offertes sont indépendantes. Ainsi ${\overline{\Delta b_{\mathrm{m}} \Delta b_{\mathrm{m}}}}^{\prime}=\overline{\Delta b}_{\mathrm{m}} \overline{\Delta b}_{\mathrm{m}}{ }^{\prime}=0$ pour $m \neq m^{\prime}$ et $\overline{\Delta b_{\mathrm{m}} \Delta b_{\mathrm{m}}{ }^{\prime}}=\overline{\Delta b_{\mathrm{m}}^{2}}$ pour $m=m^{\prime}$, c'est à dire aussi pour $\boldsymbol{r}_{\mathrm{m}}=\boldsymbol{r}_{\mathrm{m}}$. L'Eq. 6.6, s'écrit finalement :

$$
\overline{\langle\tilde{b}(0) \tilde{b}(\boldsymbol{r})\rangle}=\left\langle\tilde{b}_{\mathrm{c}}(0) \tilde{b}_{\mathrm{c}}(\boldsymbol{r})\right\rangle+\sum_{\mathrm{m}} \overline{\Delta b_{\mathrm{m}}^{2}} \frac{\delta(\boldsymbol{r})}{V} .
$$


Dont la transformée de Fourier donne :

$$
\tilde{\sigma}(\boldsymbol{q})=\operatorname{TF}\left\langle\tilde{b}_{\mathrm{c}}(0) \tilde{b}_{\mathrm{c}}(\boldsymbol{r})\right\rangle+\frac{1}{V} \sum_{\mathrm{m}} \underbrace{\overline{\Delta b_{\mathrm{m}}^{2}}}_{\left(\sigma_{\mathrm{inc}}\right)_{\mathrm{m}}} .
$$

Le premier terme correspond à la diffusion dite « cohérente » et le second à la diffusion dite « incohérente ». Ce dernier est rigoureusement indépendant de $\boldsymbol{q}$ et uniforme sur $4 \pi$ sr. La variance $\left(\sigma_{\text {inc }}\right)_{\mathrm{m}}$ est appelée « section efficace différentielle de diffusion incohérente » de l'atome $m$.

Pour un atome, les variations de sa longueur de diffusion selon son spin ou son isotope sont statistiquement indépendantes. Les variances $\left(\sigma_{\text {inc }}^{\text {spin }}\right)_{\mathrm{m}}$ et $\left(\sigma_{\text {inc }}^{\text {iso }}\right)_{\mathrm{m}}$ correspondantes s'ajoutent :

$$
\left(\sigma_{\text {inc }}\right)_{\mathrm{m}}=\left(\sigma_{\text {inc }}^{\mathrm{spin}}\right)_{\mathrm{m}}+\left(\sigma_{\text {inc }}^{\text {iso }}\right)_{\mathrm{m}} \text {. }
$$

La contribution $\sigma_{\text {inc }}^{\text {spin }}$ due au spin nucléaire est tabulée pour tous les isotopes [9]. Le tableau 2 donne les valeurs des sections efficaces cohérente et incohérente d'isotopes principaux. Notons la valeur particulièrement élevée de la section efficace incohérente du proton ${ }^{1} \mathrm{H}$.

Tableau 2. Longueurs de diffusion cohérente et section efficace différentielle de diffusion cohérente et incohérente des éléments les plus courants d'après réf. [9].

\begin{tabular}{|l|c|c|c|c|c|}
\hline & ${ }^{12} \mathrm{C}$ & ${ }^{16} \mathrm{O}$ & ${ }^{14} \mathrm{~N}$ & ${ }^{1} \mathrm{H}$ & ${ }^{2} \mathrm{H}$ \\
\hline$b_{\mathrm{c}}\left(10^{-12} \mathrm{~cm}\right)$ & 0.665 & 0.580 & 0.937 & -0.374 & 0.667 \\
$\sigma_{\mathrm{c}}\left(10^{-24} \mathrm{~cm}^{2} / \mathrm{sr}\right)$ & 0.442 & 0.337 & 0.878 & 0.126 & 0.444 \\
$\sigma_{\text {inc }}^{\text {spin }}\left(10^{-24} \mathrm{~cm}^{2} / \mathrm{sr}\right)$ & 0 & 0 & 0.040 & $\mathbf{6 . 3 9}$ & 0.163 \\
\hline
\end{tabular}

La contribution $\sigma_{\text {inc }}^{\text {iso }}$ due à la distribution aléatoire des différents isotopes dépend de la façon dont l'échantillon est préparé. Pour un échantillon de composition isotopique naturelle, la plupart des éléments chimiques sont représentés par un isotope largement majoritaire et $\sigma_{\text {inc }}^{\text {iso }}$ est très faible.

\subsection{Diffusion incohérente par un mélange de composés chimiques élémentaires}

Très souvent les échantillons sont faits de composés chimiques par exemple moléculaire. Pour un composé chimique donné indicé $\mathrm{n}$, la définition d'une densité de longueur de diffusion sous-entend celle d'un volume élémentaire $v_{\mathrm{n}}$ (par exemple le volume partiel de la molécule) qui est légitime si le composé est suffisamment petit par rapport à $q^{-1}$. Dans ce cas, la longueur de diffusion cohérente correspondante est la somme des longueurs de diffusion moyenne de chaque atome du composé (les amplitudes des ondes diffusées par chaque atome s'ajoutent) :

$$
\left(b_{\mathrm{c}}\right)_{\mathrm{n}}=\sum_{\text {atome } \in \mathrm{n}} \bar{b}_{\text {atome }} \text {. }
$$

On peut alors définir une densité de longueur de diffusion cohérente $\left(\tilde{b}_{c}\right)_{\mathrm{n}}=\left(b_{c}\right)_{\mathrm{n}} / v_{\mathrm{n}}$. La section efficace de diffusion incohérente s'obtient par contre en additionnant les variances (les intensités diffusées s'ajoutent) :

$$
\left(\sigma_{\text {inc }}\right)_{\mathrm{n}}=\sum_{\text {atome } \in \mathrm{n}}\left(\overline{\Delta b^{2}}\right)_{\text {atome }}
$$

Soit un milieu constitué d'un ensemble de composés chimiques appartenant à $\mathcal{N}$ espèces différentes, l'ensemble occupant une fraction $\phi$ du volume total et chaque espèce une fraction $\phi_{\mathrm{n}}(\mathrm{n}=0 \cdots \mathcal{N}-1)$. 
Localement la fraction de volume occupée est :

$$
\phi(\boldsymbol{r})=\sum_{n=0}^{\mathcal{N}-1} \phi_{\mathrm{n}}(\boldsymbol{r})
$$

et la densité locale de longueur de diffusion cohérente :

$$
\tilde{b}_{\mathrm{c}}(\boldsymbol{r})=\sum_{n=0}^{\mathcal{N}-1}\left(\tilde{b}_{\mathrm{c}}\right)_{\mathrm{n}} \phi_{\mathrm{n}}(\boldsymbol{r}) .
$$

Par rapport aux équations 6.1 et 6.2, si le composé chimique n de l'échantillon est constitué de $n_{\mathrm{n}}$ unités indiscernables alors $\phi_{\mathrm{n}}(\boldsymbol{r})=n_{\mathrm{n}} v_{\mathrm{n}} \rho_{\mathrm{n}}(\boldsymbol{r})$. La composante de la diffusion incohérente due au spin nucléaire s'écrit :

$$
\tilde{\sigma}_{\text {inc }}^{\text {spin }}=\sum_{\mathrm{n}} \frac{\phi_{\mathrm{n}}}{v_{\mathrm{n}}}\left(\sigma_{\mathrm{inc}}^{\mathrm{spin}}\right)_{\mathrm{n}}
$$

et celle due à une composition isotopique aléatoire :

$$
\tilde{\sigma}_{\text {inc }}^{\text {iso }}=\sum_{\mathrm{n}}\left(\frac{\phi_{\mathrm{n}}}{v_{\mathrm{n}}} \sum_{\text {iso } \in \mathrm{n}} x_{\text {iso }}\left(b_{\text {iso }}-\left(b_{c}\right)_{\mathrm{n}}\right)^{2}\right)
$$

où l'indice $\left.\right|_{\text {iso }}$ représente toutes les possibilités de composition isotopique pour le composé chimique $\mathrm{n}$ et $x_{\text {iso }}$ la proportion volumique de chacune d'elles $\left(\sum_{\text {iso }} x_{\text {iso }}=1\right)$. Dans la pratique, cet incohérent isotopique n'est important que lorsque la composition naturelle est modifiée. Par exemple pour varier le contraste, un composé chimique donné peut être utilisé sous la forme d'un mélange du composé « naturel » et du même composé dont des deutériums ont été substitués aux protons. Si certains hydrogènes du composé sont labiles (liaisons $\mathrm{O}-\mathrm{H}$ ou $\mathrm{N}-\mathrm{H}$ ) ceux-ci peuvent se répartir aléatoirement et réversiblement sur les composés chimiques ayant eux-même des hydrogènes labiles. L'incohérent isotopique n'est plus alors négligeable. Un cas typique est celui d'un mélange ${ }^{1} \mathrm{H}_{2} \mathrm{O} /{ }^{2} \mathrm{H}_{2} \mathrm{O}$ préparé avec une fraction $x$ d'eau légère. Après mélange, les molécules ${ }^{1} \mathrm{H}_{2} \mathrm{O},{ }^{2} \mathrm{H}_{2} \mathrm{O}$ et ${ }^{1} \mathrm{H}^{2} \mathrm{HO}$ sont présentes dans les proportions respectives $x^{2},(1-x)^{2}$ et $2 x(1-x)$. Connaissant $x$, on calcule aisément $\tilde{\sigma}_{\text {inc }}^{\text {iso }}$ au moyen de l'Eq. 6.18 et de la table 2 .

Finalement, cette renormalisation du diffuseur élémentaire effectuée, l'Eq.6.11 se réécrit :

$$
\tilde{\sigma}(\boldsymbol{q})=\operatorname{TF}\left\langle\tilde{b}_{\mathrm{c}}(0) \tilde{b}_{\mathrm{c}}(\boldsymbol{r})\right\rangle+\underbrace{\sum_{\mathrm{n}} \frac{\phi_{\mathrm{n}}}{v_{\mathrm{n}}}\left(\sigma_{\text {inc }}^{\text {spin }}\right)_{\mathrm{n}}}_{\tilde{\sigma}_{\text {inc }}^{\text {spin }}}+\underbrace{\sum_{\mathrm{n}}\left(\frac{\phi_{\mathrm{n}}}{v_{\mathrm{n}}} \sum_{\text {iso } \in \mathrm{n}} x_{\text {iso }}\left(b_{\text {iso }}-\left(b_{c}\right)_{\mathrm{n}}\right)^{2}\right)}_{\tilde{\sigma}_{\text {iso }}^{\text {iso }}} .
$$

Remarque : Dans le langage du neutronicien, la diffusion incohérente est due à la variance de la longueur de diffusion que présente un diffuseur élémentaire donné, lorsque cette grandeur se comporte comme une variable aléatoire. Le cas du mélange ${ }^{1} \mathrm{H}_{2} \mathrm{O} /{ }^{2} \mathrm{H}_{2} \mathrm{O}$ correspond bien à ce qu'il est convenu d'appeler « incohérent isotopique » du fait des échanges des atomes d'hydrogène. Une molécule donnée, que l'on identifierait par exemple par son atome d'oxygène, a une longueur de diffusion qui change constamment de façon aléatoire. Par contre, le cas d'un mélange de solvant naturel et deutérié dont les hydrogènes ne seraient pas labiles est en toute rigueur différent. Un tel mélange présente des fluctuations de densité de longueur de diffusion (i.e. des fluctuations de concentration) dont la longueur de corrélation est très courte (la taille d'une molécule). Pour cette raison, à petite valeur de $q$, la diffusion correspondante s'apparente à de la diffusion incohérente et est souvent qualifiée d' " incohérent de mélange ». Ce cas est traité dans la partie 6.4. 


\subsection{Fluctuations de densité}

A un milieu constitué de $\mathcal{N}$ espèces chimiques, assignons l'indice 0 au constituant majoritaire (qui est par exemple le solvant dans le cas d'une solution), on peut écrire: $\phi(\boldsymbol{r})=\phi_{0}(\boldsymbol{r})+\sum_{n=1}^{\mathcal{N}-1} \phi_{\mathrm{n}}(\boldsymbol{r})$, d'où la densité de longueur de diffusion cohérente :

$$
\tilde{b}_{\mathrm{c}}(\boldsymbol{r})=\sum_{n=1}^{\mathcal{N}-1} \tilde{b}_{\mathrm{c} n} \phi_{\mathrm{n}}(\boldsymbol{r})+\tilde{b}_{\mathrm{c} 0}\left(\phi(\boldsymbol{r})-\sum_{n=1}^{\mathcal{N}-1} \phi_{\mathrm{n}}(\boldsymbol{r})\right)=\sum_{n=1}^{\mathcal{N}-1}\left(\tilde{b}_{\mathrm{c} n}-\tilde{b}_{\mathrm{c} 0}\right) \phi_{\mathrm{n}}(\boldsymbol{r})+\tilde{b}_{\mathrm{c} 0} \phi(\boldsymbol{r}) .
$$

La différence $\left(\tilde{b}_{\mathrm{c} n}-\tilde{b}_{\mathrm{c} 0}\right)$ est appelée « densité de longueur de contraste » du constituant $\mathrm{n}$. Le terme $\tilde{c}_{\mathrm{n}}(\boldsymbol{r})=\left(\tilde{b}_{\mathrm{c} n}-\tilde{b}_{\mathrm{c} 0}\right) \phi_{\mathrm{n}}(\boldsymbol{r})$ correspond à sa contribution au contraste local $\tilde{c}(\boldsymbol{r})$. Cette grandeur et sa moyenne $\tilde{c}$ sur tout l'échantillon, s'écrivent :

$$
\tilde{c}(\boldsymbol{r})=\sum_{n=1}^{\mathcal{N}-1}\left(\tilde{b}_{\mathrm{c} n}-\tilde{b}_{\mathrm{c} 0}\right) \phi_{\mathrm{n}}(\boldsymbol{r}) \quad \text { et } \quad \tilde{c}=\sum_{n=1}^{\mathcal{N}-1}\left(\tilde{b}_{\mathrm{c} n}-\tilde{b}_{\mathrm{c} 0}\right) \phi_{\mathrm{n}} .
$$

On obtient pour la fonction de corrélation :

$$
\langle\tilde{b}(0) \tilde{b}(\boldsymbol{r})\rangle=\langle\tilde{c}(0) \tilde{c}(\boldsymbol{r})\rangle+\tilde{b}_{\mathrm{c} 0}^{2}\langle\phi(0) \phi(\boldsymbol{r})\rangle+2 \tilde{b}_{\mathrm{c} 0} \sum_{n=1}^{\mathcal{N}-1}\left(\tilde{b}_{\mathrm{c} n}-\tilde{b}_{\mathrm{c} 0}\right)\left\langle\phi_{\mathrm{n}}(0) \phi(\boldsymbol{r})\right\rangle .
$$

En notant que $\left\langle\phi_{\mathrm{n}}(0) \phi(\boldsymbol{r})\right\rangle=\phi_{\mathrm{n}} / \phi \times\langle\phi(0) \phi(\boldsymbol{r})\rangle$, on obtient :

$$
\langle\tilde{b}(0) \tilde{b}(\boldsymbol{r})\rangle=\langle\tilde{c}(0) \tilde{c}(\boldsymbol{r})\rangle+\tilde{b}_{\mathrm{c} 0}^{2}\left(1+\frac{2 \tilde{c}}{\tilde{b}_{\mathrm{c} 0} \phi}\right)\langle\phi(0) \phi(\boldsymbol{r})\rangle .
$$

Le premier terme de cette somme correspond aux différentes fonctions de corrélation de composition (ou de concentration), le deuxième aux fluctuations de la densité. Ces dernières font intervenir une distance caractéristique $a$ telle que $a^{3}$ est le volume élémentaire moyen des constituants (par exemple le volume partiel d'une molécule), distance qui est très petite comparée $1 / q$. A cette échelle $\langle\Delta \phi(0) \Delta \phi(\boldsymbol{r})\rangle \simeq$ $\left\langle\Delta \phi^{2}\right\rangle \delta(\boldsymbol{r} / a)$. Sa transformée de Fourier $\mathrm{TF}\langle\Delta \phi(0) \Delta \phi(\boldsymbol{r})\rangle \simeq a^{3}\left\langle\Delta \phi^{2}\right\rangle=\phi^{2} k T \kappa_{T}$ est une constante indépendante de $\boldsymbol{q}$ et proportionnelle à la compressibilité isotherme $\kappa_{T}$ du milieu. Finalement la transformée de Fourier de l'Eq. 6.23 donne

$$
\tilde{\sigma}(\boldsymbol{q})=\operatorname{TF}\langle\tilde{c}(0) \tilde{c}(\boldsymbol{r})\rangle+\underbrace{\tilde{b}_{\mathrm{c} 0}^{2}\left(1+\frac{2 \tilde{c}}{\tilde{b}_{\mathrm{c} 0} \phi}\right) \phi^{2} k T \kappa_{T}}_{\tilde{\sigma}_{\kappa}} .
$$

Remarque : la fraction moyenne $\phi$ vaut 1 dans le cas d'un milieu dense mais est inférieure pour un matériau poreux ou une mousse par exemple.

\section{4 « Incohérent » de mélange}

Les différentes méthodes de variation de contraste amènent souvent l'expérimentateur à utiliser un mélange de solvants de même nature chimique, mais dont l'un a une composition isotopique naturelle et l'autre modifiée par substitution de deutériums aux protons. Lorsque les atomes d'hydrogène des molécules ne sont pas labiles, les neutrons «voient » un mélange de deux constituant. Soit $\tilde{b}_{c 0 \mathrm{H}}$ et $\tilde{b}_{c 0 \mathrm{D}}$ les densités de longueur de diffusion cohérente des solvants naturel et deutérié. La fonction de corrélation $\langle\tilde{c}(0) \tilde{c}(\boldsymbol{r})\rangle$ s'écrit :

$$
\langle\tilde{c}(0) \tilde{c}(\boldsymbol{r})\rangle=\left(\tilde{b}_{c 0 \mathrm{D}}-\tilde{b}_{c 0 \mathrm{H}}\right)^{2}\left\langle\phi_{\mathrm{D}}(0) \phi_{\mathrm{D}}(\boldsymbol{r})\right\rangle,
$$

où $\phi_{\mathrm{D}}$ est la fraction volumique du solvant deutérié. Les deux types de molécules de solvant étant de même nature chimique, aucune interaction privilégiée (et donc aucune corrélation) n'est attendue entre 
molécules deutériées. D'où $\left\langle\phi_{\mathrm{D}}(0) \phi_{\mathrm{D}}(\boldsymbol{r})\right\rangle=\left\langle\Delta \phi_{\mathrm{D}}^{2}\right\rangle \delta\left(\boldsymbol{r} / v_{0}^{1 / 3}\right)$, où $v_{0}$ est le volume moléculaire partiel du solvant (supposé identique dans les deux cas). Finalement on obtient :

$$
\langle\tilde{c}(0) \tilde{c}(\boldsymbol{r})\rangle=\left(\tilde{b}_{c 0 \mathrm{D}}-\tilde{b}_{c 0 \mathrm{H}}\right)^{2} \phi_{\mathrm{D}}\left(1-\phi_{\mathrm{D}}\right) \delta\left(\boldsymbol{r} / v_{0}^{1 / 3}\right)
$$

et sa transformée de Fourier :

$$
\mathrm{TF}\langle\tilde{c}(0) \tilde{c}(\boldsymbol{r})\rangle=\underbrace{\left(\tilde{b}_{c 0 \mathrm{D}}-\tilde{b}_{c 0 \mathrm{H}}\right)^{2} \phi_{\mathrm{D}}\left(1-\phi_{\mathrm{D}}\right) v_{0}}_{\tilde{\sigma}_{\text {mél }}} .
$$

La section efficace différentielle par unité de volume $\tilde{\sigma}_{\text {mél }}$ est indépendante de $q$ et s'apparente à une diffusion incohérente qui peut participer au bruit de fond inhérent à l'échantillon.

\subsection{Comment évaluer le bruit de fond inhérent à l'échantillon ?}

Les équations 6.19 et 6.24 permettent d'écrire la section efficace différentielle de diffusion comme la somme d'une fonction $\tilde{s}(\boldsymbol{q})=\mathrm{TF}\langle\tilde{c}(0) \tilde{c}(\boldsymbol{r})\rangle$ correspondant à une diffusion centrale et de termes constants qui participent au bruit de fond inhérent à l'échantillon :

$$
\tilde{\sigma}(\boldsymbol{q})=\tilde{s}(\boldsymbol{q})+\underbrace{\tilde{\sigma}_{\text {inc }}^{\text {spin }}+\tilde{\sigma}_{\text {inc }}^{\text {iso }}+\tilde{\sigma}_{\kappa}+\tilde{\sigma}_{\text {mél }}}_{\tilde{\sigma}_{\mathrm{BdF}}} .
$$

Le bruit de fond $\tilde{\sigma}_{\mathrm{BdF}}$ pourrait en principe être calculé connaissant la composition chimique de l'échantillon. Toutefois, les arguments développés dans la partie 5.1 relative à la valeur absolue de l'intensité diffusée par un échantillon de référence, restent valables ici. Le bruit de fond inhérent à l'échantillon doit être mesuré.

La fonction $\tilde{s}(\boldsymbol{q})$ caractéristique des corrélations de composition dans l'échantillon est une fonction globalement décroissante qui tend vers 0 à grand vecteur de diffusion. La méthode la plus simple pour estimer $\tilde{\sigma}_{\mathrm{BdF}}$ consisterait donc à mesurer $\tilde{\sigma}(\boldsymbol{q})$ jusqu'à des grandes valeurs de $q$ afin d'atteindre son asymptote $\tilde{\sigma}_{\mathrm{BdF}}$. Toutefois, $\tilde{s}(\boldsymbol{q})$ peut ne pas décroître assez rapidement pour que le régime asymptotique soit observable sur un appareil de petits angles. D'ailleurs des mesures à plus grands angles sortiraient du domaine de validité des Eq. 6.24 et 6.27 (à très grand $\boldsymbol{q}$ la partie cohérente de $\tilde{\sigma}_{\mathrm{BdF}}$ n'est plus constante).

L'estimation du bruit de fond d'un échantillon passe alors par la préparation d'échantillons dont le spectre de diffusion $\tilde{\sigma}_{\text {tém }}$ serait indépendant de $q$ (donc facilement mesurable) et témoignerait du bruit de fond $\tilde{\sigma}_{\mathrm{BdF}}$ inhérent à l'échantillon. Par exemple lorsque l'échantillon est une solution, un témoin peut être préparé sur la base du même solvant en ajustant le rapport proton/deutérium pour obtenir $\tilde{\sigma}_{\text {tém }}=\tilde{\sigma}_{\mathrm{BdF}}$. Cette préparation est guidée par le calcul des valeurs théoriques de $\tilde{\sigma}_{\text {tém }}$ et $\tilde{\sigma}_{\mathrm{BdF}}$ au moyen des Eq. 6.19, 6.24 et 6.27. Généralement lors d'une série d'expériences, plusieurs échantillons sont étudiés chacun avec des bruits de fond différents. Le nombre de témoins préparés (et donc le temps de mesure) peut être réduit par interpolation des mesures. Si on note $X$ une variable de laquelle dépendent à la fois $\tilde{\sigma}_{\text {tém }}$ et $\tilde{\sigma}_{\mathrm{BdF}}$. Soit $\tilde{\sigma}_{\text {tém }}^{(i)}$ la section efficace mesurée pour le témoin $i$ et $\mathscr{C}$ la courbe d'interpolation des couples $\left(X^{(i)}, \sigma_{\text {tém }}^{(i)}\right)$. Le bruit de fond de l'échantillon est donné par le point de la courbe $\mathscr{C}$ dont l'abscisse $X$ est celle de l'échantillon. Le tableau 3 compare le calcul des différentes contributions qui interviennent dans la diffusion de l'eau et du cyclohexane pris comme exemple. Dans le cas de l'eau légère, la diffusion incohérente domine, tandis que pour l'eau lourde, diffusion cohérente et incohérente sont du même ordre de grandeur. Des résultats comparables sont attendus entre tout milieu dont l'un a une composition isotopique naturelle en hydrogène et l'autre dont des deutériums ont été substitués aux protons. La variable $X$ à utiliser pour interpoler les valeurs $\sigma_{\text {tém }}^{(i)}$ dépend duquel de ces deux cas relève l'échantillon.

1. Si l'incohérent domine: l'approximation consiste à négliger la partie cohérente $\tilde{\sigma}_{\kappa}$ du bruit de fond de l'échantillon. Dans l'Eq. 6.28, la diffusion centrale $\tilde{s}(\boldsymbol{q})$ est cantonnée à un angle solide très petit comparé aux $4 \pi$ sr de la diffusion uniforme du bruit de fond incohérent. La diffusion 
Tableau 3. Tableau comparatif des termes intervenant dans la section efficace différentielle par unité de volume de l'eau et du cyclohexane. Pour l'eau à température ambiante $k T \kappa_{T}=1.88 \times 10^{-24} \mathrm{~cm}^{3}$ et $v_{0}=30.0 \times 10^{-24} \mathrm{~cm}^{3}$, pour le cyclohexane $k T \kappa_{T}=4.63 \times 10^{-24} \mathrm{~cm}^{3}$ et $v_{0}=179 \times 10^{-24} \mathrm{~cm}^{3}[10]$.

\begin{tabular}{|c|c|c|c|c|c|c|}
\hline & $\phi_{\mathrm{H}} / \phi_{\mathrm{D}}$ & $\begin{array}{c}\tilde{\sigma}_{\text {inc }}^{\text {spin }} \\
\mathrm{cm}^{-1} / \mathrm{sr}\end{array}$ & $\begin{array}{c}\tilde{\sigma}_{\text {inc }}^{\text {iso }} \\
\mathrm{cm}^{-1} / \mathrm{sr}\end{array}$ & $\begin{array}{c}\tilde{\sigma}_{\kappa} \\
\mathrm{cm}^{-1} / \mathrm{sr}\end{array}$ & $\begin{array}{c}\tilde{\sigma}_{\text {mél }} \\
\mathrm{cm}^{-1} / \mathrm{sr}\end{array}$ & $\begin{array}{c}\tilde{\sigma} \text { (total) } \\
\mathrm{cm}^{-1} / \mathrm{sr}\end{array}$ \\
\hline \hline eau & $1 / 0$ & 0.427 & - & $5.9 \times 10^{-5}$ & - & 0.427 \\
& $0 / 1$ & 0.011 & - & $7.7 \times 10^{-3}$ & - & 0.019 \\
& $0.5 / 0.5$ & 0.219 & 0.036 & $1.6 \times 10^{-3}$ & - & 0.257 \\
\hline \hline cyclohexane & $1 / 0$ & 0.428 & - & $3.6 \times 10^{-5}$ & - & 0.428 \\
& $0 / 1$ & 0.011 & - & 0.021 & - & 0.032 \\
& $0.5 / 0.5$ & 0.214 & - & $9.0 \times 10^{-6}$ & 0.218 & 0.432 \\
\hline
\end{tabular}

totale intégrée sur $4 \pi$ sr est donc pratiquement identique pour l'échantillon et son témoin ce qui implique des transmissions identiques. La variable $X$ sera donc la valeur de la transmission.

2. Si les fluctuations de densité ne sont pas négligeables: dans ce cas les témoins n'ont pas une diffusion uniforme sur $4 \pi$ sr et en particulier diffuse plus à grand angle (facteur de structure d'un liquide). Or la transmission reflète la diffusion totale qui ne varie pas forcément de la même façon que celle mesurée à petit angle. Cela implique que la variable correcte d'interpolation n'est plus la transmission. Toutefois, toutes les composantes du bruit de fond de l'échantillon (Eq. 6.19, 6.24 et 6.27) peuvent s'exprimer en fonction de la quantité $x_{H}$ de protons de l'échantillon. Cette variable sera celle utilisée pour $X$. Ce cas est nettement moins favorable que le précédent et demande un contrôle très précis de la quantité de protons introduite dans les échantillons témoins ce qui est particulièrement difficile dans le cas de solutions aqueuses.

\subsection{Quelle épaisseur pour l'échantillon?}

Un dernier point pratique qui suscite des questions concerne l'épaisseur des échantillons. L'Eq. 3.1 montre que l'intensité diffusée varie comme $I \propto Z \exp (-\mu Z)$. Ce produit passe par un maximum pour

$$
Z=\frac{1}{\mu}=\Lambda
$$

où $\Lambda$ est le libre parcours moyen d'un neutron dans l'échantillon (c'est à dire la distance moyenne entre deux collisions avec un atome de l'échantillon, collisions conduisant à une diffusion). Cela correspond à une transmission $\mathcal{T}=\exp (-\mu Z)=1 / e \simeq 0.37$. Il est clair qu'augmenter l'épaisseur de l'échantillon jusqu'à s'approcher de cette valeur optimale de la transmission, occasionne des diffusions multiples. Deux cas sont à considérer. Si la diffusion incohérente prédomine, la diffusion simple étant déjà isotrope, la diffusion multiple ne pose pas de problème en dehors de ceux évoqués dans la partie 5 relative à l'étalonnage du spectromètre (cf. la remarque à la fin de la partie 3.1). Par contre, si la diffusion est principalement centrale et cohérente il est préférable de s'approcher d'une transmission de 1 . Un bon critère pour identifier rapidement si la diffusion multiple est uniquement incohérente consiste à vérifier rapidement que l'intensité mesurée est bien proportionnelle à l'épaisseur.

\section{Conclusion}

Ce cours tente de répondre aux questions concrètes que se pose un expérimentateur en abordant la diffusion de neutrons aux petits angles. Ces questions ont d'abord été les miennes. Formé en premier lieu à la diffusion de la lumière, la cohérence du faisceau de neutrons ou l'approximation élastique sont des questions qui ont surgi très vite. Puis sont venues toutes les questions très pratiques liées au réglage 
du spectromètre, aux mesures à effectuées ou au traitement des données. La littérature est pour le moins très discrète sur ces problèmes sans doute trop « triviaux ». Personnellement la plupart des réponses me sont venues plutôt par « infusion » lente dans la culture du laboratoire (merci à Loïc Auvray, Annie Brûlet, Patrick Calmettes, Jean-Pierre Cotton, José Teixeira). J'ai donc compilé ici tous ces points et essayé de produire un outil pour accélérer ce processus d'apprentissage.

Une question liée à l'instrument qui n'a pas été abordée est celle de l'analyse des données en relation avec la résolution instrumentale. La première difficulté de cette analyse est commune aux techniques fournissant une information dans l'espace réciproque: un spectre mesuré dans un intervalle fini de vecteur de diffusion permet rarement d'accéder à une information univoque dans l'espace direct (problème inverse). La seconde difficulté est qu'en diffusion de neutrons aux petits angles la résolution est fonction du vecteur de diffusion. Un spectre n'est donc pas à proprement parlé un simple produit de convolution de la densité spectrale de puissance de l'échantillon par une fonction d'appareil. La solution de ce problème passe nécessairement par un modèle paramétré de l'échantillon dont on calcule la section efficace de diffusion théorique qui une fois « convoluée » par la résolution est comparée à la mesure, permettant ainsi une optimisation des paramètres du modèle $[11,12]$. Pour ces questions liées en particulier à la modélisation, le lecteur est également renvoyé au cours de Luc Belloni dans ce même volume.

\section{Références}

[1] D. Lairez. Résolution d'un spectromètre de diffusion de neutrons aux petits angles. J. Phys. IV France, 9:67-81, 1999.

[2] D.F.R. Mildner and J.M. Carpenter. Optimization of the experimental resolution for small-angle scattering. J. Appl. Cryst., 17:249-256, 1984.

[3] J.-P. Cotton, D. Decker, H. Benoît, B. Farnoux, J. Higgins, G. Jannink, R. Ober, C. Picot, and J. des Cloizeaux. Conformation of polymer chain in the bulk. Macromolecules, 7:863, 1974.

[4] J.-P. Cotton. Initial data treatment. In P. Linder and Th. Zemb, editors, Neutron, X-ray and light scattering., chapter 2, pages 19-31. North Holland Publishing, 1991.

[5] A. Brûlet, D. Lairez, A. Lapp, and J.-P. Cotton. Improvement of data treatment in small angle neutron scattering. J. Appl. Cryst., 40:165-177, 2007.

[6] P. Strunz, J. Saroun, U. Keiderling, A. Wiedenmann, and R. Przenioslo. General formula for determination of cross-section from measured sans intensities. J. Appl. Cryst., 33:829-833, 2000.

[7] M. Nelkin. Scattering of slow neutrons by water. Phys. Rev., 119(2):741-746, Jul 1960.

[8] Y. Edura and N. Morishima. Cold and thermal neutron scattering in liquid water: cross-section model and dynamics of water molecules. Nuclear Instr. Meth. Phys. Res. A, 534:531-543, 2004.

[9] Varley F. Sears. Neutron scattering lengths and cross sections. Neutron News, 3(3):26-37, 1992.

[10] D. R. Lide, editor. Handbook of Chemistry and Physics. CRC Press, 76th edition, 1995-1996.

[11] O. Glatter. The inverse scattering problem in small-angle scattering. In P. Linder and Th. Zemb, editors, Neutrons, X-rays and light: scattering methods applied to soft condensed matter., chapter 4, pages 73-102. Elsevier Science B.V., 2002.

[12] O. Glatter. Fourier transformation and deconvolution. In P. Linder and Th. Zemb, editors, Neutrons, X-rays and light: scattering methods applied to soft condensed matter, chapter 5, pages 73-124. Elsevier Science B.V., 2002. 